\title{
THEORY OF REDUCTION FOR ARITHMETICAL EQUIVALENCE. II $\left({ }^{1}\right)$
}

\author{
BY \\ HERMANN WEYL
}

1. Introduction. Lattices over the unit lattice. Given $n$ linearly independent vectors $\delta_{1}, \cdots, \delta_{n}$ in an $n$-dimensional vector space $E^{n}$, the formula

$$
\mathfrak{x}=y_{1} \delta_{1}+\cdots+y_{n} \delta_{n}
$$

yields all vectors of the space $E^{n}$ or of a lattice $\&$ in $E^{n}$ if the coordinates $y_{i}$ range over all real numbers or all integers, respectively. We take the viewpoint that the lattice $\mathfrak{R}$ is given but the choice of its basis arbitrary. The several bases are connected with one another by unimodular transformations. If $f(\mathfrak{x})$ is a gauge function assigning a "length" $f(\mathfrak{x})$ to each vector $\mathfrak{x}$ the problem of reduction requires normalization of the lattice basis in terms of the given $f$. A solution is sought for all possible gauge functions or at least for some important class. The most significant class is obtained by running $f^{2}$ over all positive quadratic forms.

Following in Dirichlet's and Hermite's footsteps, Minkowski developed such a method of reduction for quadratic forms and established the decisive facts about it. In R1 I approached the same problem in that geometric way which Minkowski had initiated but then abandoned for unknown reasons.

The question may be put in a slightly different form. All linear mappings of $E^{n}$ carrying $\mathbb{R}$ into itself carry $f(\mathfrak{x})$ into equivalent gauge functions. The task is to pick out by a universal rule in each class of equivalent gauge functions one particular $f(\mathfrak{x})$ which is called the reduced function of its class. Let $R_{0}, R, \mathcal{C}$ in the future denote the fields of all rational, real and complex numbers, respectively. Complex numbers are written in the form $\xi=x_{0}+x_{1} i$ $\left(x_{0}, x_{1}\right.$ real $)$. It is convenient to insert between the full vector space and the lattice $\mathfrak{R}$, the set $E_{0}^{n}$ of all vectors (1) with rational coefficients $y_{i}$, a set which we describe as an $n$-dimensional vector space over $\mathbb{R}_{0}$. Crystallography has found this advisable in distinguishing between the macroscopic and atomistic symmetries of a crystal, and in the theory of algebraic numbers one puts the field before the ring of its integers.

Let a lattice $\mathfrak{R}$ in $E_{0}^{n}$ be given. With respect to any basis $\delta_{1}, \cdots, \delta_{n}$ of $E_{0}^{n}$, formula (1), the function $f(\mathfrak{x})$ is represented by a function $g\left(y_{1}, \cdots, y_{n}\right)$ and the lattice $\&$ by a "numerical lattice" $\Lambda$ whose vectors are $n$-uples $\left(y_{1}, \cdots, y_{n}\right)$

Presented to the Society, January 1, 1941; received by the editors December 11, 1940.

(1) The first part, which appeared under the same title in these Transactions, vol. 48 (1940), pp. $126-164$, is cited as $\mathrm{R} 1$. 
of rational numbers. (Only if $\delta_{1}, \cdots, b_{n}$ is a true basis of $\mathfrak{R}$ will $\Lambda$ be the unit lattice I whose elements are the $n$-uples of integers.) Hence $f(\mathfrak{x})$ with respect to $\mathfrak{R}$ is represented by $g / \Lambda$. All representations $g / \Lambda$ of $f(\mathfrak{x}) / \mathfrak{R}$ are equivalent, i.e., they arise from one another by linear transformations of the coordinates with rational coefficients. In each class of equivalent $g / \Lambda$ we are to pick one individual, the "reduced" $g / \Lambda$. Suppose we have succeeded in doing this by some universal rule. We then have to select, for each $\Lambda$ that may occur in a reduced $g / \Lambda$, a definite basis $\delta_{1}{ }^{*}, \cdots, \delta_{n}{ }^{*}$ in terms of which $\mathfrak{R}$ is represented by $\Lambda$. The equation

$$
f^{*}(\mathfrak{x})=g\left(y_{1}, \cdots, y_{n}\right) \text { for } \mathfrak{x}=y_{1} \delta_{1}{ }^{*}+\cdots+y_{n}{\delta_{n}}^{*}
$$

then defines the reduced gauge function $f^{*}$ in its class. By the first step of reducing $g / \Lambda$ no essential progress has been made unless the lattices $\Lambda$ which may occur in a reduced $g / \Lambda$ are limited to a finite number of possibilities. For only then is the selection of a basis $\delta_{1}{ }^{*}, \cdots, b_{n}{ }^{*}$ for each of these $\Lambda$ essentially simpler than the original problem.

The Dirichlet-Hermite-Minkowski method of reduction by admitting only bases $b_{1}, \cdots, b_{n}$ of $\mathfrak{R}$ always represents $\mathfrak{R}$ by the one lattice $\Lambda=I$, the unit lattice. Thus it provides the ideal solution. Minkowski's construction of consecutive shortest distances in the lattice

$$
f\left(\delta_{1}\right)=M_{1}, \cdots, f\left(\grave{D}_{n}\right)=M_{n}
$$

(for which he obtains the inequality $M_{1} \cdots M_{n} V \leqq 2^{n}$ ) falls under our more general scheme. That theorem which he describes as indicating a certain "Oekonomie der Strahldistancen" states exactly that there is only an $a$ priori limited number of possibilities for $\Lambda$ with which to count in a reduced $g / \Lambda$. In R1 I carried the first method over to those other fields and quasi-fields which have not more than one infinite prime spot, and I found that it works only under the hypothesis that the class number for ideals is 1 . Simultaneously Siegel observed that the rougher second method, by which incidentally Minkowski had proved that the class number of positive quadratic forms with integral coefficients and a given discriminant is finite, operates without this restrictive hypothesis $\left({ }^{2}\right)$. I add the remark that an argument making no use of the bases of a lattice need not even assume their existence. In an algebraic number field $\mathcal{F}$ we consider any "order" [f] ; in general there are several classes of lattices belonging to this order. The theory is limited neither to the principal class nor to the principal order. Following a suggestion by Siegel, $P$. Humbert generalized the investigation of quadratic forms to an arbitrary algebraic number field $\mathcal{F}$ with several infinite prime spots $\left({ }^{2}\right)$. No doubt the whole problem thereby loses much of its simplicity. But once upon this track one ought to include the quaternions and thus deal also with those noncom-

(2) See P. Humbert, Commentarii Mathematici Helvetici, vol. 12 (1939-1940), pp. 263-306. 
mutative division algebras of finite degree over $R_{0}$ for which the concept of infinite prime spots goes through. I resume here the rougher method of reduction with these further generalizations by the same geometric approach as in R1. I am not only interested in the fact that certain numbers are finite; I wish to ascertain reasonably low explicit upper bounds for them. The geometric method yields good results in this regard.

Before concluding this introduction I remind the reader of some simple facts about lattices in $E_{0}^{n}$. A vector $\mathfrak{x}$ in $E_{0}^{n}$ is defined as an $n$-uple $\left(x_{1}, \cdots, x_{n}\right)$ of rational numbers. The unit vectors $e_{k}=\left(e_{1 k}, \cdots, e_{n k}\right)$ are the columns of the unit matrix $\left\|e_{i k}\right\|$. The word lattice means any set of vectors such that $\mathfrak{a}-\mathfrak{b}$ is contained in the set every time $\mathfrak{a}$ and $\mathfrak{b}$ are. We assume that the lattice is $n$-dimensional, i.e., contains $n$ linearly independent vectors; and discrete, i.e., we require that for any given positive integer $q$ there are not more than a finite number of lattice vectors satisfying the inequalities

$$
\left|x_{1}\right| \leqq q, \cdots,\left|x_{n}\right| \leqq q .
$$

From now on the term lattice refers only to discrete lattices which have the full dimensionality of their vector space. By a familiar argument one shows that one can find $n$ linearly independent vectors $\mathfrak{l}_{1}, \ldots, \mathfrak{l}_{n}$ in a given lattice $\mathfrak{R}$ such that every lattice vector

$$
\mathfrak{x}=u_{1} \mathfrak{l}_{1}+\cdots+\dot{u}_{n} \mathfrak{l}_{n}
$$

has integral components $u_{i}$. By the same construction one adapts the basis $\mathfrak{l}_{1}, \cdots, \mathfrak{l}_{n}$ of any lattice $\Lambda$ containing the unit lattice $I$ to the basis $\mathfrak{e}_{1}, \cdots, \mathfrak{e}_{n}$ of I:

$$
\begin{aligned}
\mathrm{e}_{1} & =c_{1} \mathfrak{l}_{1}, \\
\mathrm{e}_{2} & =c_{21} \mathrm{l}_{1}+c_{2} \mathfrak{l}_{2}, \\
\cdot & \cdot \cdots \cdot \cdots \cdot \cdot \\
\mathrm{e}_{n} & =c_{n 1} \mathrm{l}_{1}+\cdots+c_{n, n-1} \mathfrak{l}_{n-1}+c_{n} \mathfrak{l}_{n} .
\end{aligned}
$$

The integers $c_{k}$ are positive and the integral skew coefficients $c_{k i}(i<k)$ may be normalized by

$$
0 \leqq c_{k i}<c_{i} \quad(k=i+1, \cdots, n) ;
$$

then $\left(\mathfrak{l}_{1}, \cdots, \mathfrak{l}_{n}\right)$ is uniquely determined. The index $j=[\Lambda: \mathrm{I}]$, i.e., the number of vectors in $\Lambda$ which are incongruent modulo $I$, equals $c_{1} \cdots c_{n}$. Let $\Lambda^{(k)}$ denote the part of $\Lambda$ lying in the linear subspace $x_{k+1}=\cdots=x_{n}=0$. The index $j_{k}=\left[\Lambda^{(k)}: I^{(k)}\right]$ equals $c_{1} \cdots c_{k}$. Hence these two lemmas:

LEMMA 1. $j_{k}$ is a divisor of $j_{h}$ for $k<h$.

Lemma 2. The number $h_{n}(j)$ of different lattices $\Lambda$ over $\mathrm{I}$ of given index $j=[\Lambda: \mathrm{I}]$ is finite. 
Indeed, it equals the sum

$$
\sum c_{1}^{n-1} c_{2}^{n-2} \cdots c_{n}^{0}
$$

extended over all factorizations $c_{1} c_{2} \cdots c_{n}=j$ of $j$. (Incidentally, the numbers $h_{n}(j)$ for $j=1,2, \cdots$ have as their generating function the Dirichlet series

$$
\sum_{j=1}^{\infty} h_{n}(j) \cdot j^{-s}=\zeta(s) \zeta(s-1) \cdots \zeta(s-n+1)
$$

convergent in the half-plane $R s>n$.)

2. Vector space and lattice over an algebraic field. Let $\mathcal{F}$ be any field of finite degree $f$ over $R_{0}$. By carefully putting all factors in their proper places we shall see to it that all arguments and formulas in this and the following two sections remain valid for any division algebra, whether commutative or not, of finite degree over $R_{0}$. We choose a basis $\sigma_{1}, \cdots, \sigma_{f}$ of $\mathcal{F} / R_{0}$ so that each number $\xi$ of $\mathcal{F}$ is uniquely represented by

$$
\xi=x_{1} \sigma_{1}+\cdots+x_{f} \sigma_{f}
$$

( $x_{a}$ rational).

Any $n$-uple $\left(\xi_{1}, \cdots, \xi_{n}\right)$ of numbers $\xi_{i}$ in $\mathcal{F}$ is a vector of the $n$-dimensional vector space $E^{n}$ over $\mathcal{F}$. The fundamental operations are addition of two vectors, $\mathfrak{x}+\mathfrak{x}^{\prime}$, and multiplication $\delta \mathfrak{x}$ of a vector $\mathfrak{x}$ by a number $\delta$ (the numerical factor always in front of the vector!). Thus we may write

$$
\mathfrak{x}=\left(\xi_{1}, \cdots, \xi_{n}\right)=\xi_{1} e_{1}+\cdots+\xi_{n} e_{n} .
$$

$k$ linearly independent vectors $\delta_{1}, \cdots, \delta_{k}$ span a linear subspace $\left[\delta_{1}, \cdots, \delta_{k}\right]$ consisting of all vectors of the form $\eta_{1} \delta_{1}+\cdots+\eta_{k} \delta_{k}$. Any $n$ linearly independent vectors $\delta_{1}, \cdots, \grave{b}_{n}$ form a basis of $E^{n} / \mathcal{F}$ in terms of which each vector is uniquely expressible as

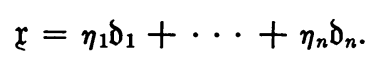

The original coordinates $\xi_{i}$ are connected with the $\eta_{i}$ by that nonsingular linear transformation $D$,

$$
\xi_{i}=\sum_{k} \eta_{k} \delta_{i k}
$$

whose matrix $\left\|\delta_{i k}\right\|$ has for its columns the vectors $\delta_{k}=\left(\delta_{1 k}, \cdots, \delta_{n k}\right)$.

Expressing each component $\xi_{i}$ in terms of the basis $\sigma$ of $\mathcal{F}$,

$$
\xi_{i}=x_{i 1} \sigma_{1}+\cdots+x_{i f} \sigma_{f},
$$

we identify $E^{n} / \mathcal{F}$ with the (nf)-dimensional vector space $E_{0}^{n f}$ over $\mathcal{R}_{0}$. The rational numbers $x_{i a}$ are the coordinates of $\mathfrak{r}$ with respect to the basis $\sigma_{a} e_{i}$. One has to distinguish between linear dependence in $\mathcal{F}$ and in $R_{0}$.

We suppose we are given a lattice $\mathfrak{l}$ in $E_{0}^{n f}$. It will have a basis $\mathfrak{l}_{\mu}$ 
$(\mu=1, \cdots, n f)$ in terms of which each vector $\mathfrak{x}$ of $\mathfrak{R}$,

$$
\mathfrak{x}=\sum_{\mu} u_{\mu} \mathfrak{l}_{\mu}
$$

has rational integral components $u_{\mu}$. A number $\delta$ of $\mathcal{F}$ is said to be a multiplier of $\mathfrak{R}$ if the operation $\mathfrak{x} \rightarrow \delta \mathfrak{x}$ carries each lattice vector $\mathfrak{r}$ into a lattice vector $\delta \mathfrak{r}$. The multipliers of $\&$ form an order [f]. This assertion is meant to imply the following four properties $\left({ }^{3}\right)$ :

$1^{\circ}$. The number 1 is in [f].

$2^{\circ}$. [ $\left.\mathcal{F}\right]$ is a ring.

$3^{\circ}$. Any given number $\delta$ in $\mathcal{F}$ may be multiplied by a positive rational integer $m$ such that $m \delta$ is in [f] .

$4^{\circ}$. Each number in $[\mathcal{F}]$ is an integer.

$1^{\circ}$ and $2^{\circ}$ are evident. To prove $3^{\circ}$ and $4^{\circ}$ we write

$$
\delta \mathfrak{l}_{\mu}=\sum_{\nu} d_{\nu \mu} \mathfrak{l}_{\nu}
$$

If $\delta$ is any number and $m$ a common denominator of the coefficients $d_{\mu \nu}$, then $m \delta$ is a multiplier. If $\delta$ happens to be a multiplier, then the $d_{\mu \nu}$ are rational integers and $\delta$ satisfies the equation

$$
\left|\delta e_{\nu \mu}-d_{\nu \mu}\right|=0 \text {. }
$$

In the same manner as for the "principal order" consisting of all integers of $\mathcal{F}$ one proves $\left({ }^{4}\right)$ that any order $[\mathcal{F}]$ is a discrete $f$-dimensional lattice in the $f$-dimensional vector space $\mathcal{F} / R_{0}$, and hence has a basis $\sigma_{1}, \cdots, \sigma_{f}$ in terms of which every number $\xi$ of [f] appears in the form (2) with rational integral coefficients $x_{i}$.

The transformation $D$, (4), maps $\&$ upon a lattice $\Lambda$ : If $\mathfrak{x}=\left(\xi_{1}, \cdots, \xi_{n}\right)$ is in $\mathfrak{R}$, then $\left(\eta_{1}, \cdots, \eta_{n}\right)$ is in $\Lambda$, and vice versa. We call two lattices equivalent and admit them to the same class if one is carried into the other by a nonsingular transformation $D$. The lattices $\Lambda$ of one class express a given lattice $\mathbb{R}$ in terms of different bases $\left(b_{1}, \cdots, \grave{b}_{n}\right)$ of $E^{n} / \mathcal{F}$. Obviously two equivalent lattices have the same multipliers.

A lattice $\mathfrak{l}$ is said to belong to the order $[\mathcal{F}]$ if every number of that order is a multiplier of $\mathfrak{R}$. (For $n=1$ this notion coincides with that of an ideal in [F] , and our classes of lattices with the classes of ideals.) Given an order [f], the $n$-uples $\left(\xi_{1}, \cdots, \xi_{n}\right)$ of numbers $\xi_{i}$ in [f] form a lattice I which belongs to the order [f]; we call it the unit lattice for $[\mathcal{F}]$. The lattices belonging to a given order [f] are distributed over a number of classes, the class of $I$ being the principal class.

$\left({ }^{3}\right)$ Notion and name are due to Dedekind. Hilbert in his Zahlbericht introduced the word "ring" for this purpose, but since ring has no'v acquired a wider meaning I revert, in agreement with such authorities as Artin and Chevalley, to Dedekind's terminology.

(4) Cf. H. Weyl, Algebraic Theory of Numbers, Princeton, 1940, pp. 145-146. 
Let $\sigma_{1}, \cdots, \sigma_{f}$ be a basis of [f] and $\mathfrak{l}_{\mu}(\mu=1, \cdots, n f)$ a basis of $\Lambda$. If $\Lambda$ contains $I$ the vectors $\sigma_{a} \mathfrak{e}_{k}$, which span I, are linear combinations of the $\mathfrak{l}_{\mu}$ with integral rational coefficients, and their absolute determinant, i.e., the absolute determinant of the transformation connecting the coordinates $u_{\mu}$ with the $x_{k a}(k=1, \cdots, n ; a=1, \cdots, f)$ is the index $j=[\Lambda: \mathrm{I}]$.

Those vectors $\left(\xi_{1}, \cdots, \xi_{n}\right)$ in $\Lambda$ for which $\xi_{k+1}=\cdots=\xi_{n}=0$ form a lattice $\Lambda^{(k)}$ in the $k$-dimensional space $E^{k} / \mathcal{F}$ with the coordinates $\xi_{1}, \cdots, \xi_{k}$. Considering $\Lambda$ as a lattice in $E_{0}^{n f}$ and using the arrangement

$$
x_{11}, \cdots, x_{1 f} ; x_{21}, \cdots, x_{2 f} ; \cdots
$$

of the coordinates in $E_{0}^{n f}$ one can apply Lemma 1 to $k f$ and $(k+1) f$ instead of $k$ and $h$ and thus one derives a corresponding proposition in $\mathcal{F}$ instead of $R_{0}$ :

LEMma 3. In the row of indices

$$
j_{k}=\left[\Lambda^{(k)}: \mathrm{I}^{(k)}\right] \quad(k=1, \cdots, n)
$$

each number is a divisor of its successor.

The set of vectors $\left(\xi_{1}, \cdots, \xi_{n}\right)$ in $\Lambda$ outside $\left[e_{1}, \cdots, e_{k-1}\right]$, i.e., for which $\left(\xi_{k}, \cdots, \xi_{n}\right) \neq(0, \cdots, 0)$, will be denoted $\Lambda_{k}$. Thus $\Lambda_{k}$ and $\Lambda^{(k-1)}$ are complements in $\Lambda$.

We have seen that the number of different lattices $\Lambda$ over a given lattice I with a given index $[\Lambda: \mathrm{I}]=j$ is finite, namely $h_{n f}(j)$. More exactly, one finds by the same argument that the number $H_{f}\left(j_{1}, \cdots, j_{n}\right)$ of different lattices $\Lambda$ over I with given indices (6) has as its generating function the Dirichlet series of $n$ variables $s_{1}, \cdots, s_{n}$ :

$$
\begin{aligned}
\mathrm{Z}_{f}\left(s_{1}+s_{2}+\cdots+s_{n}-n f\right) \cdot \mathbf{Z}_{f}\left(s_{2}+\cdots\right. & \left.+s_{n}-(n-1) f\right) \cdots \mathrm{Z}_{f}\left(s_{n}-f\right) \\
& =\sum_{j_{1}, \cdots, j_{n}} H_{f}\left(j_{1}, \cdots, j_{n}\right) \cdot j_{1}^{-s_{1}} \cdots j_{n}^{-s_{n}}
\end{aligned}
$$

where

$$
\mathrm{Z}_{f}(s)=\zeta(s+1) \cdots \zeta(s+f)
$$

Hence $a$ fortiori:

LEMMA 4. We have found upper bounds for the number of lattices $\Lambda$ belonging to a given order [f] which contain the unit vectors $\mathrm{e}_{1}, \cdots, \mathrm{e}_{n}$ and hence the unit lattice I for [F] and which, moreover, have either a given index $j=[\Lambda: \mathrm{I}]$ or a given row of indices $j_{1}, \cdots, j_{n}$.

3. Preliminaries about reduction. Suppose an order [f] and a basis $\sigma_{1}, \cdots, \sigma_{f}$ of $[\mathcal{f}]$ to be given. We consider a real-valued function

$$
f(\mathfrak{x})=f\left(\xi_{1}, \cdots, \xi_{n}\right)
$$

which depends on a variable vector $\mathfrak{x}$ in $E^{n} / \mathcal{F}$ and is positive except for $\mathfrak{x}=0$, and we assume: 
(io) For each positive $q$ one can ascertain a positive $q^{\prime}$ such that the inequality $f(\mathfrak{x})<q$ implies the $n f$ inequalities

$$
\left|x_{i a}\right|<q^{\prime} \quad(i=1, \cdots, n ; a=1, \cdots, f)
$$

for the components $x_{i a}$ of the $\xi_{i}$.

Let $\mathfrak{R}$ be a lattice belonging to the fixed order [f]. $n$ vectors $\delta_{1}, \cdots, \delta_{n}$ of $\mathfrak{Z}$ which are linearly independent with respect to $\mathcal{F}$ constitute a semi-basis of $\mathfrak{R}$. Because of the discreteness of $\mathfrak{R}$ there is but a finite number of vectors $\mathfrak{x}$ in $\mathfrak{R}$ satisfying the inequalities (7). Hence Minkowski's construction of consecutive minima of $f$ in $\mathfrak{R}$ is applicable. It yields a semi-basis $\mathfrak{D}_{1}, \cdots, \mathfrak{b}_{n}$ of $\mathfrak{R}$ such that

$$
f(\mathfrak{x}) \geqq f\left(\mathfrak{b}_{k}\right)=M_{k}
$$

for every vector $\mathfrak{x}$ in $\mathfrak{R}$ outside $\left[\delta_{1}, \ldots, \delta_{k-1}\right]$ (reduced semi-basis). Obviously

$$
M_{1} \leqq M_{2} \leqq \cdots \leqq M_{n}
$$

The mapping

$$
\mathfrak{x}=\eta_{1} \delta_{1}+\cdots+\eta_{n} \delta_{n} \rightarrow\left(\eta_{1}, \cdots, \eta_{n}\right)
$$

carries $f(\mathfrak{x})$ into a function $g\left(\eta_{1}, \cdots, \eta_{n}\right)$ and $\mathfrak{R}$ into a lattice $\Lambda$ which contains the unit lattice $I$ for [f]. The function $g\left(\xi_{1}, \cdots, \xi_{n}\right)$ is reduced with respect to $\Lambda$, i.e.,

$$
g\left(\xi_{1}, \cdots, \xi_{n}\right) \geqq g\left(e_{1 k}, \cdots, e_{n k}\right)
$$

whenever $\left(\xi_{1}, \cdots, \xi_{n}\right)$ is in $\Lambda_{k}$.

The $M_{k}$ are uniquely determined by $f$ and $R$; the situation is somewhat less favorable for $\delta_{1}, \cdots, \delta_{n}$. Suppose $\delta_{1}^{\prime}, \cdots, \delta_{n}^{\prime}$ is another set constructed according to our prescription. If $M_{k}$ is actually lower than $M_{k+1}$ then $\left[\delta_{1}^{\prime}, \ldots, \delta_{k}^{\prime}\right]=\left[\delta_{1}, \cdots, \delta_{k}\right]$. (Analogues of Theorems 8 and 9 in R1.)

Being given $n$ real numbers $p_{k}$,

$$
1 \leqq p_{1} \leqq \cdots \leqq p_{n},
$$

we say that the semi-basis $\delta_{1}^{\prime}, \cdots, b_{n}^{\prime}$ of $\mathbb{R}$ has the property $B\left(p_{1}, \cdots, p_{n}\right)$ if

$$
f(\mathfrak{x}) \geqq \frac{1}{p_{k}} f\left(\boldsymbol{D}_{k}^{\prime}\right)
$$

for any vector $\mathfrak{x}$ in $\mathfrak{l}$ outside $\left[\mathfrak{b}_{1}^{\prime}, \ldots, \boldsymbol{b}_{k-1}^{\prime}\right]$. Accordingly we ascribe the property $B\left(p_{1}, \cdots, p_{n}\right)$ to a function $g\left(\xi_{1}, \cdots, \xi_{n}\right)$ in conjunction with a lattice $\Lambda$ over $I$ if

$$
g\left(\xi_{1}, \cdots, \xi_{n}\right) \geqq \frac{1}{p_{k}} \cdot g\left(e_{1 k}, \cdots, e_{n k}\right)
$$

whenever $\left(\xi_{1}, \cdots, \xi_{n}\right)$ is in $\Lambda_{k}$. 
If $b_{k}^{\prime}$ is a semi-basis of $\mathfrak{Q}$ with the property $B\left(p_{1}, \cdots, p_{n}\right)$, then

$$
M_{k}^{\prime}=f\left(\delta_{k}^{\prime}\right) \leqq p_{k} M_{k} .
$$

(Analogue of Theorem $8_{p}$.) Indeed, let $\delta_{1}, \cdots, \delta_{n}$ be a reduced semi-basis of $\mathfrak{R}, f\left(\boldsymbol{b}_{k}\right)=M_{k}$. At least one of the $k$ linearly independent vectors $\boldsymbol{D}_{1}, \cdots, \boldsymbol{D}_{k}$, say $\delta_{i}$, lies outside $\left[\delta_{1}^{\prime}, \ldots, \delta_{k-1}^{\prime}\right]$; hence

or

$$
f\left(\delta_{i}\right) \geqq \frac{1}{p_{k}} \cdot f\left(\grave{D}_{k}^{\prime}\right)
$$

$$
M_{k}^{\prime} \leqq p_{k} M_{i} \leqq p_{k} M_{k}
$$

With the same notations I maintain that $\left[\delta_{1}^{\prime}, \ldots, \delta_{k}^{\prime}\right]=\left[\delta_{1}, \cdots, \delta_{k}\right]$ provided $M_{k+1}>p_{k} M_{k}$. (Analogue of Theorem $9_{p}$.) Proof: Suppose that one of the vectors $\delta_{1}^{\prime}, \cdots, \delta_{k}^{\prime}$, say $\delta_{i}^{\prime}$, is not in $\left[\delta_{1}, \cdots, \delta_{k}\right]$. Then

$$
f\left(b_{i}^{\prime}\right) \geqq M_{k+1} \text {. }
$$

Vice versa, if all $k$ numbers $M_{1}^{\prime}, \cdots, M_{k}^{\prime}$ are less than $M_{k+1}$ then $\delta_{1}^{\prime}, \cdots, \delta_{k}^{\prime}$ lie in $\left[\delta_{1}, \cdots, \delta_{k}\right]$. The observation that $M_{i}^{\prime} \leqq p_{i} M_{i} \leqq p_{k} M_{k}$ finishes the proof.

The notation of properly reduced bases depends on a given multiplicative group $U$ of numbers $\epsilon$ in $\mathcal{F}$ and deals with functions $f$ which satisfy the further condition:

(iii $) \quad f(\epsilon \mathfrak{x})=f(\mathfrak{x})(\epsilon$ in $U)$.

The semi-basis $b_{1}, \cdots, b_{n}$ of $\mathfrak{R}$ is said to be properly reduced provided the inequality

$$
f(\mathfrak{x})>f\left(\mathfrak{d}_{k}\right)
$$

holds with the sign $>$ for any vector $\mathfrak{x}$ of $\mathfrak{R}$ outside $\left[\mathfrak{b}_{1}, \cdots, \mathfrak{\delta}_{k-1}\right]$ except for the vectors of the special form

$$
\mathfrak{x}=\epsilon \boldsymbol{D}_{k}
$$

Accordingly $g\left(\xi_{1}, \cdots, \xi_{n}\right)$ is properly reduced with respect to the lattice $\Lambda$ over I if

$$
g\left(\xi_{1}, \cdots, \xi_{n}\right)>g\left(e_{1 k}, \cdots, e_{n k}\right)
$$

for all vectors $\left(\xi_{1}, \cdots, \xi_{n}\right)$ in $\Lambda_{k}$ except the special vectors

$$
\epsilon\left(e_{1 k}, \cdots, e_{n k}\right)
$$

With $\delta_{k}$ the vectors

$$
\delta_{k}^{\prime}=\epsilon_{k} \delta_{k}
$$

form a reduced semi-basis of $\mathfrak{R}$ under the sole assumption that they lie in $\mathfrak{R}$. 
Because $\mathfrak{x}=\epsilon_{k} \mathfrak{b}_{k}$ satisfies

$$
f(\mathfrak{x})=f\left(\mathfrak{b}_{k}\right), \quad \text { a fortiori } f(\mathfrak{x}) \leqq f\left(\mathfrak{b}_{k}\right),
$$

there is then, according to ( $\left.i_{0}\right)$, only a finite number of possibilities for $\epsilon_{k}$. We set

$$
\eta_{1} \delta_{1}+\cdots+\eta_{n} \delta_{n}=\mathfrak{x}=\eta_{1}^{\prime} \delta_{1}^{\prime}+\cdots+\eta_{n}^{\prime} \delta_{n}^{\prime}
$$

and denote by $\Lambda, \Lambda^{\prime}$ the corresponding images of $\mathfrak{R}$ :

$$
\left(\eta_{1}, \cdots, \eta_{n}\right) \text { in } \Lambda \cdot \rightleftarrows \cdot \mathfrak{x} \quad \text { in } \mathfrak{R} \cdot \rightleftarrows \cdot\left(\eta_{1}^{\prime}, \cdots, \eta_{n}^{\prime}\right) \text { in } \Lambda^{\prime} .
$$

The "special transformation"

$$
\eta_{k}=\eta_{k}^{\prime} \epsilon_{k}
$$

carries $\Lambda$ into $\Lambda^{\prime}$. We count in the same family any two lattices $\Lambda$ and $\Lambda^{\prime}$ arising from each other by such a special transformation. Given the lattice $\Lambda$ over I there is only a finite number of special transformations such that the transformed lattice $\Lambda^{\prime}$ also contains I. In particular, the group $\left\{J_{\Lambda}\right\}$ of all special transformations $J_{\Delta}$ leaving $\Lambda$ invariant is finite. If $h$ is its degree, one has $\epsilon_{k}^{n}=1(k=1, \cdots, n)$ for each $J_{\Lambda}$; hence the $\epsilon_{k}$ are roots of unity in $\mathcal{F}$. The roots of unity in a field $\mathcal{F}$ form a finite cyclic group; in particular, if $\mathcal{F}$ has at least one real spot, the only such roots are \pm 1 . (However, in noncommutative division algebras the group of the roots of unity is, generally speaking, neither Abelian nor finite.)

The simple argument in R1, p. 136, shows:

If $\delta_{1}, \cdots, \delta_{n}$ is a properly reduced semi-basis and $\delta_{1}^{\prime}, \cdots, \delta_{n}^{\prime}$ any semibasis of $\mathfrak{R}$, then the sequence of the values $f\left(\delta_{1}\right), \cdots, f\left(\delta_{n}\right)$ is lower than $f\left(\boldsymbol{b}_{1}^{\prime}\right), \cdots, f\left(\mathfrak{b}_{n}^{\prime}\right)$. If $\boldsymbol{b}_{1}^{\prime}, \cdots, \mathfrak{b}_{n}^{\prime}$ is reduced and $\mathfrak{b}_{1}, \cdots, \boldsymbol{b}_{n}$ properly reduced. then

$$
\delta_{1}^{\prime}=\epsilon_{1} \delta_{1}, \cdots, \delta_{n}^{\prime}=\epsilon_{n} \delta_{n}
$$

$\left(\epsilon_{i}\right.$ in $\left.U\right)$.

4. Extension to the ground field $R$. Minkowski's inequality. So far the function $f(\mathfrak{x})$ has been defined merely for the vectors in the space $E^{n} / \mathcal{F}$. In order to introduce geometry we assign to the variables $x_{a}$ in (2) arbitrary real values:

$$
\xi^{*}=x_{1} \sigma_{1}+\cdots+x_{f} \sigma_{f}
$$

Sticking to the multiplication table of the basic elements $\sigma_{a}$, we thus extend $\mathcal{F} / \mathbb{R}_{0}$ to a commutative algebra $\mathcal{F}^{*}$ over $R$. But only in the two cases treated in $R 1$, where $\mathcal{F}$ is $R_{0}$ itself or an imaginary quadratic field over $R_{0}$, is $\mathcal{F}^{*}$ again a field. In general it is not. However, any $n$-uple $\mathfrak{r}^{*}=\left(\xi_{1}^{*}, \cdots, \xi_{n}^{*}\right)$ of elements $\xi_{i}^{*}$ in $\mathcal{F}^{*}$ may be considered as a vector in an $(n f)$-dimensional vector space $E^{n f}$ over $R$ with the real coordinates $x_{i a}$ : 


$$
\xi_{i}^{*}=x_{i 1} \sigma_{1}+\cdots+x_{i j} \sigma_{f} .
$$

We now assume $f\left(\mathfrak{t}^{*}\right)$ to be a gauge function, i.e., a continuous real-valued function in this space, having the following properties:

(i) $f\left(\mathfrak{x}^{*}\right)>0$ except for $\mathfrak{x}^{*}=0$.

(ii) $f\left(t \mathfrak{r}^{*}\right)=|t| \cdot f\left(\mathfrak{x}^{*}\right)$ for any real factor $t$.

(iii) $f\left(\mathfrak{x}_{1}^{*}+\mathfrak{x}_{2}^{*}\right) \leqq f\left(\mathfrak{x}_{1}^{*}\right)+f\left(\mathfrak{x}_{2}^{*}\right)$.

The gauge body

$$
K: \quad f\left(\xi_{1}^{*}, \cdots, \xi_{n}^{*}\right)<1
$$

and also the solid $q K$ defined by $f\left(\xi_{1}^{*}, \cdots, \xi_{n}^{*}\right)<q$ are bounded; hence postulate $\left(\mathrm{i}_{0}\right)$ of the previous section is fulfilled. Let $V^{*}$ be the volume of $K$ computed in terms of the coordinates $x_{i a}$.

Again we fix an order [f] and a basis $\sigma_{1}, \cdots, \sigma_{f}$ of [f] . Let $\Lambda$ be a lattice belonging to this order and containing the unit lattice $I$ for [F] and let $f\left(\xi_{1}, \cdots, \xi_{n}\right)$ be reduced with respect to $\Lambda$. The volume of $K$ in terms of the coordinates $u_{\mu}$ as introduced by (5), i.e., measured against the fundamental parallelepiped of $\Lambda$, equals $V^{*}$. [ $\left.\Lambda: I\right]$. Hence by the simple argument explained in R1, p. 140, Minkowski's second inequality leads to this formula holding for a gauge function $f\left(\xi_{1}^{*}, \cdots, \xi_{n}^{*}\right)$ which is reduced with respect to $\Lambda$ :

$$
\left(M_{1} \cdots M_{n}\right)^{f} \cdot V^{*}[\Lambda: \mathrm{I}] \leqq 2^{n f}
$$

where

$$
M_{k}=f\left(e_{1 k}, \cdots, e_{n k}\right) .
$$

5. Splitting. The number of reduced lattices is finite. Up to now everything has worked for a division algebra of degree $f$ over $R_{0}$ just as well as for a field $\mathcal{F}$. Further progress depends on the structure of $\mathcal{F}^{*}$. If $\mathcal{F}$ is a field, then $\mathcal{F}^{*}$ is isomorphic to the direct sum of a number of components $R$ and $\mathcal{C}$. We first study this case.

The decomposition of $\mathcal{F}^{*}$ is brought about by conjugation. One knows that $\mathcal{F} / \mathcal{R}_{0}$ has a determining number $\theta$ whose powers $1, \theta, \cdots, \theta^{\prime-1}$ constitute a basis for $\mathcal{F}$. The number $\theta$ satisfies an irreducible equation in $R_{0}$ of degree $f$. Let $\theta^{\alpha}$ and $\theta^{\beta}, \bar{\theta}^{\beta}$ (or in one row : $\theta^{(1)}, \cdots, \theta^{(f)}$ ) denote its $r$ real and $s$ pairs of complex conjugate roots. They define the $f$ conjugations

$$
\xi \rightarrow \xi^{\alpha} ; \quad \xi \rightarrow \xi^{\beta}, \quad \xi \rightarrow \bar{\xi}^{\beta}
$$

each of which projects $\mathcal{F}$ isomorphically into $R$ or $\mathcal{C}$. We use the notations

$$
\xi^{\alpha}=x^{\alpha}, \quad \xi^{\beta}=x_{0}^{\beta}+i x_{1}^{\beta} \quad\left(\bar{\xi}^{\beta}=x_{0}^{\beta}-i x_{1}^{\beta}\right)
$$

and call the $r+s$ numbers $\xi^{\alpha}, \xi^{\beta}$ the splits, and the $f$ real numbers $x^{\alpha} ; x_{0}^{\beta}, x_{1}^{\beta}$ 
the splitting coordinates of $\xi$. The same applies to any element $\xi^{*}$ of $\mathcal{F}^{*}$. The product $\zeta^{*}=\xi^{*} \eta^{*}$ has the splits

$$
\zeta^{\alpha}=\xi^{\alpha} \eta^{\alpha}, \quad \zeta^{\beta}=\xi^{\beta} \eta^{\beta} .
$$

The arithmetician speaks of the different values of the indices $\alpha$ and $\beta$ as the $r$ real and $s$ imaginary (infinite prime) spots of $\mathcal{F}$; for the sake of brevity we often drop the adjectives in parentheses. If a definite arrangement is desired, we write $\alpha=\alpha_{1}, \cdots, \alpha_{r} ; \beta=\beta_{1}, \cdots, \beta_{\text {s. }}$

The splitting coordinates $x^{\alpha} ; x_{0}^{\beta}, x_{1}^{\beta}$ are connected with the components $x_{1}, \cdots, x_{f}$ of $\xi^{*}$ by the linear substitution

$$
\Sigma=\left\|\sigma_{1}, \cdots, \sigma_{f}\right\|
$$

where in the symbol on the right side each term stands for the column of its splitting coordinates (in a definite arrangement). The splitting of $\mathcal{F}^{*}$ into $r$ components $R$ and $s$ components $\mathcal{C}$ is established as soon as it is certain that the absolute determinant

$$
\Delta=\text { abs. }\left|\sigma_{1}, \cdots, \sigma_{f}\right|
$$

of the matrix $\Sigma$ is different from zero. For the particular basis $1, \theta, \cdots, \theta^{f-1}$ one sees that $(-2 i)^{s} \cdot \Delta$ is the Vandermonde determinant of $\theta^{(1)}, \cdots, \theta^{(f)}$, and hence indeed $\Delta \neq 0$. This fact carries over to any basis $\sigma_{1}, \cdots, \sigma_{f}$ of $\mathcal{F}$.

The number in $\mathcal{F}^{*}$ with the splitting coordinates $x^{\alpha} ; x_{0}^{\beta},-x_{1}^{\beta}$ is denoted by $\bar{\xi}^{*}$. As absolute value $\left|\xi^{*}\right|$ we introduce the greatest of the $r+s$ numbers $\left|\xi^{\alpha}\right|,\left|\xi^{\beta}\right|$. One could agree on other definitions, but this one is most convenient for our future applications. What usually is called a unit in $\mathcal{F}$ is a number of $\mathcal{F}$ which is a unity at all finite prime spots. None but the infinite prime spots matter for our investigation; hence we take the liberty of using the term "unit" for those numbers $\epsilon$ of $\mathcal{F}$ which are unities at all infinite prime spots, i.e., for which the $r+s$ equations $\left|\epsilon^{\alpha}\right|=1,\left|\epsilon^{\beta}\right|=1$ hold.

For any element $\delta^{*}$ of $\mathcal{F}^{*}$ one introduces the real matrix $\left\|d_{a b}\right\|$ of the linear operation $\xi^{*} \rightarrow \xi^{*} \delta^{*}$ in $\mathcal{F}^{*}$ :

$$
x_{a} \rightarrow \sum_{b} d_{a b} x_{b} \quad\left(\sigma_{a} \delta^{*}=\sum_{b} d_{b a} \sigma_{b}\right)
$$

and its characteristic polynomial

$$
\left|t e_{a b}-d_{a b}\right|=t^{f}-d_{1} t^{f-1}+\cdots \pm d_{f} .
$$

$d_{1}$ and $d_{f}$ are called trace (tr) and norm $(\mathrm{Nm})$, respectively. In terms of the splitting coordinates our operation of multiplication splits into the transformations

$$
x^{\alpha} \rightarrow x^{\alpha} d^{\alpha} ; \quad \xi^{\beta} \rightarrow \xi^{\beta} \delta^{\beta},
$$


each corresponding to a real or imaginary spot $\alpha$ or $\beta$. Of course, $\xi^{\beta} \rightarrow \xi^{\beta} \delta^{\beta}$ stands for

$$
\stackrel{\beta}{x_{0}} \rightarrow \stackrel{\beta}{\beta} x_{0}^{\beta} d_{0}^{\beta}-x_{1}^{\beta} d_{1}^{\beta}, \quad x_{1}^{\beta} \rightarrow x_{0}^{\beta} d_{1}^{\beta}+x_{1}^{\beta} d_{0}^{\beta} .
$$

Hence

$$
\begin{aligned}
\operatorname{tr}\left(\delta^{*}\right) & =\sum_{\alpha} d^{\alpha}+2 \sum_{\beta} d_{0}^{\beta}, \\
\operatorname{Nm}\left(\delta^{*}\right) & =\prod_{\alpha} d^{\alpha} \cdot \prod_{\beta}\left\{\left(d_{0}^{\beta}\right)^{2}+\left(d_{1}^{\beta}\right)^{2}\right\} .
\end{aligned}
$$

If $\delta^{*}=\delta$ is in $\mathcal{F}$ the $d_{a b}$ are rational numbers. For a unit $\epsilon$ in $\mathcal{F}$ our formulas show that the determinant $\mathrm{Nm}(\epsilon)$ of the transformation $\xi^{*} \rightarrow \xi^{*} \epsilon$ is of absolute value 1 and hence as a rational number equal to \pm 1 .

Considering the trace $\operatorname{tr}\left(\xi^{2}\right)$ one readily verifies that $\left(2^{\delta} \Delta\right)^{2}$ is rational for any basis $\sigma_{1}, \cdots, \sigma_{f}$ and especially a rational integer for a basis of an order $[\mathcal{F}]$.

The transformation (4) in $E^{n f}$,

$$
\xi_{i}^{*}=\sum_{k} \eta_{k}^{*} \delta_{i k}
$$

$\left(\delta_{i k}\right.$ numbers in $\left.\mathcal{F}\right)$

splits into the components

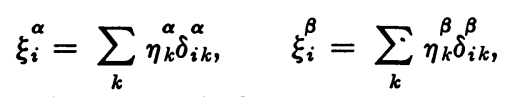

each $\alpha$-component involving $n$, each $\beta$-component $2 n$ real variables:

$$
\xi_{k}^{\alpha}=x_{k}^{\alpha} ; \quad \xi_{k}^{\beta}=\stackrel{\beta}{x_{k 0}}+i x_{k 1}^{\beta} .
$$

How closely can one approximate an element $\xi^{*}$ of $\mathcal{F}^{*}$ by a number $\gamma$ of our order [f] with the basis $\sigma_{1}, \cdots, \sigma_{f}$ ? For an appropriate $\gamma$ in [f] the real components $x_{a}^{\prime}$ of $\xi^{*}-\gamma$,

$$
\xi^{*}-\gamma=x_{1}^{\prime} \sigma_{1}+\cdots+x_{f}^{\prime} \sigma_{f},
$$

will satisfy the inequalities $\left|x_{a}^{\prime}\right| \leqq \frac{1}{2}$, and thus

where

$$
\left|\xi^{*}-\gamma\right| \leqq \rho
$$

$$
\rho=\frac{1}{2} \cdot \max _{\alpha, \beta}\left(\left|\sigma_{1}^{\alpha}\right|+\cdots+\left|\sigma_{f}^{\alpha}\right|,\left|\sigma_{1}^{\beta}\right|+\cdots+\left|\sigma_{f}^{\beta}\right|\right) .
$$

The "circles" of radius $\rho$ around all numbers $\gamma$ of $[\mathcal{F}]$ cover the whole $\mathcal{F}^{*}$. (Such a radius was denoted by the letter $r$ in R1, which now serves a different purpose.)

Let us now return to the situation explained at the end of the previous section and let $V$ be the volume of $K$ computed in terms of the splitting coordinates of $\xi_{1}^{*}, \cdots, \xi_{n}^{*}$. Then $V=V^{*} / \Delta^{n}$. Moreover we observe that 
$f\left(\xi_{1}^{*}, \cdots, \xi_{k}^{*}, 0, \cdots, 0\right)$ is reduced with respect to the lattice $\Lambda^{(k)}$, and denoting by $V_{k}$ the volume of the solid

$$
f\left(\xi_{1}^{*}, \cdots, \xi_{k}^{*}, 0, \cdots, 0\right)<1
$$

in $E^{k f}$ computed in terms of the splitting coordinates of $\xi_{1}^{*}, \cdots, \xi_{\mathfrak{k}}^{*}$, we obtain these fundamental inequalities for $M_{k}=f\left(e_{1 k}, \cdots, e_{n k}\right)$ :

Theorem I. For a reduced $f\left(\xi_{1}, \cdots, \xi_{n}\right) / \Lambda$ one has

$$
\left(M_{1} \cdots M_{n}\right)^{f} \cdot V[\Lambda: \mathrm{I}] \leqq\left(2^{f} \Delta\right)^{n},
$$

more generally

$$
\left(M_{1} \cdots M_{k}\right)^{f} \cdot V_{k}\left[\Lambda^{(k)}: \mathrm{I}^{(k)}\right] \leqq\left(2^{f} \Delta\right)^{k} .
$$

At this point we introduce the further assumption:

(ii*) $f\left(\tau^{*} \mathfrak{r}^{*}\right) \leqq\left|\tau^{*}\right| \cdot f\left(\mathfrak{r}^{*}\right)$

$\left(\tau^{*}\right.$ any element of $\left.\mathcal{F}^{*}\right)$,

and henceforth the term "gauge function" is to be taken in this restricted sense. Following Minkowski's own argument, we then prove

THEOREM II. For a reduced $f\left(\xi_{1}, \cdots, \xi_{n}\right) / \Lambda$ one always has

$$
j=[\Lambda: \mathrm{I}] \leqq(n f) !\left(\frac{4}{\pi}\right)^{n s} \cdot\left(\frac{\Delta}{f !}\right)^{n}
$$

and more generally

$$
j_{k}=\left[\Lambda^{(k)}: \mathrm{I}^{(k)}\right] \leqq(k f) !\left(\frac{4}{\pi}\right)^{k s}\left(\frac{\Delta}{f !}\right)^{k} \quad(k=1, \cdots, n) .
$$

Hence in any class of lattices belonging to the order [f] there is always a lattice $\Lambda$ which contains I and satisfies (13) and $\left(13_{k}\right)$. Together with Lemma 2 this proves $\left({ }^{5}\right)$ :

THEOREM III. The number of classes of lattices belonging to a given order $[\mathcal{F}]$ is finite.

(5) This theorem is well known. We are concerned only with those lattices $\Lambda$ over I which are in the class of $\mathfrak{R}$, but as our bounds (13) or the sharper bounds (35) depend on the order rather than on the special class it seemed worth while to mention Theorem III in passing. For a commutative field $\mathcal{F}$ and its principal order [f] E. Steinitz, Mathematische Annalen, vol. 71 (1912), pp. 328-354, and vol. 72 (1912), pp. 297-345, proved that the number for classes of any $n$ is the same as for $n=1$, namely equal to the number of classes of ideals. See also I. Schur, Mathematische Annalen, vol. 71 (1912), pp. 355-367; W. Franz, Journal für die reine und angewandte Mathematik, vol. 171 (1934), pp. 149-161; C. Chevalley, L'Arithmétique dans les Algèbres de Matrices, Actualités Scientifiques et Industrielles, no. 323, 1936, in particular Theorems 3,7 and 8 . 
(The proposition implies the corresponding one about classes of ideals.) Any gauge function will do for the proof, for instance

$$
f\left(\xi_{1}^{*}, \cdots, \xi_{n}^{*}\right)=\left|\xi_{1}^{*}\right|+\cdots+\left|\xi_{n}^{*}\right| .
$$

We shall soon see that much better upper bounds for the number of classes are obtained by using for $f^{2}$ the trace of a positive Hermitian form. However, our present Theorem II goes far beyond Theorem III because it deals with any gauge function $f$ in conjunction with a lattice rather than with lattices alone.

Proof. Observe that the "octahedron"

$$
\left|\xi_{1}^{*}\right|+\cdots+\left|\xi_{n}^{*}\right|<1
$$

contains no vector of $\Lambda$ except the zero vector. Hence owing to Minkowski's chief inequality we find this upper bound for its volume $W$ :

$$
W j \leqq\left(2^{f} \Delta\right)^{n} \text {. }
$$

Let $\left(\xi_{1}, \cdots, \xi_{n}\right)$ be a vector in $\Lambda$ and $\xi_{k}$ be the last nonvanishing one among its coordinates $\xi_{i}$. Then by the definition of reduction

$$
f\left(\xi_{1}, \cdots, \xi_{n}\right) \geqq M_{k}=f\left(e_{1 k}, \cdots, e_{n k}\right) .
$$

On the other hand the assumptions (iii) and (ii*) imply

$$
\begin{aligned}
f\left(\xi_{1} e_{1}+\cdots+\xi_{n} e_{n}\right) & \leqq M_{1}\left|\xi_{1}\right|+\cdots+M_{n}\left|\xi_{n}\right| \\
& =M_{1}\left|\xi_{1}\right|+\cdots+M_{k}\left|\xi_{k}\right| .
\end{aligned}
$$

Because of (8) the relations (14) and (15) are incompatible unless

$$
\left|\xi_{1}\right|+\cdots+\left|\xi_{n}\right|=\left|\xi_{1}\right|+\cdots+\left|\xi_{k}\right| \geqq 1 .
$$

We base our computation of $W$ upon the following general remark about gauge functions $f$ in an $n$-dimensional vector space over $R$. If $V$ is the volume of the gauge body $K: f(\mathfrak{x})<1$, then the integral $\int$ of $e^{-f}$ over the whole space equals $n ! V$. One simply evaluates the integral by decomposing the space into the infinitely thin shells

$$
q \leqq f(\mathfrak{x})<q+d q
$$

and thus finds

$$
\int=V \cdot \int_{0}^{\infty} e^{-q} \cdot n q^{n-1} d q=n ! V .
$$

Applying this remark to the gauge function $\left|\xi_{1}^{*}\right|+\cdots+\left|\xi_{n}^{*}\right|$ in our (nf)dimensional vector space and to the gauge function $\left|\xi^{*}\right|$ in the $f$-dimensional space $\mathcal{F}^{*}$, one gets this double value for $\int$ :

$$
(n f) ! W=(f ! w)^{n} \text {, }
$$


$w$ being the volume of the "cylinder" defined by

$$
\left|\xi^{*}\right|<1, \quad \text { or by }\left|x^{\alpha}\right|<1, \quad\left(x_{0}^{\beta}\right)^{2}+\left(x_{1}^{\beta}\right)^{2}<1 .
$$

Therefore $w=2^{r} \pi^{s}$.

(ii*) entails the property (ii ${ }_{0}$ ) of $\S 3$, provided $U$ is the group of units in our sense. From now on we shall abide by this convention and interpret the term "properly reduced" accordingly. Then the transformation $\xi^{*} \rightarrow \xi^{*} \cdot \epsilon$ ( $\epsilon$ in $U$ ) and hence every special transformation (10) has the determinant \pm 1 and thus the indices $j_{k}$ for two lattices $\Lambda$ and $\Lambda^{\prime}$ over $I$ which are in the same family coincide: $j_{k}=j_{k}^{\prime}$ for $k=1, \cdots, n$.

The values $\gamma^{*}$ of a Hermitian form in $\mathcal{F}^{*}$,

$$
\gamma^{*}\left(\mathfrak{x}^{*}\right)=\sum_{i, k} \xi_{i}^{*} \gamma_{i k}^{*} \bar{\xi}_{k}^{*} \quad\left(\begin{array}{c}
* \\
\gamma_{k i}^{*}
\end{array}=\vec{\gamma}_{i k}^{*}\right)
$$

are totally real in the sense that $\bar{\gamma}^{*}=\gamma^{*}$, or that even the $\beta$-splits $\gamma^{\beta}=g_{0}^{\beta}+i g_{1}^{\beta}=g^{\beta}$ of $\gamma^{*}$ are real. What such a Hermitian form does is to associate a quadratic form $\left\{g_{k x}^{\alpha}\right\}$ with each real spot $\alpha$ and a Hermitian form $\left\{\gamma_{i k}^{\beta}\right\}$ with every imaginary spot $\beta$. The splits of $\gamma^{*}\left(\mathfrak{r}^{*}\right)$ are

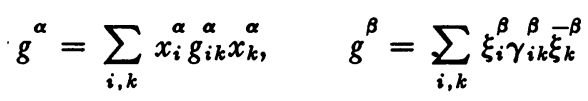

where $x_{i}^{\alpha}=\xi_{i}^{\alpha}$ and $\xi_{i}^{\beta}$ are the splits of $\xi_{i}^{*}$. The form $\gamma^{*}\left(\mathfrak{x}^{*}\right)$ is said to be positive if each of the $r$ quadratic forms $\left\{g_{k i}^{\alpha}\right\}$ and each of the $s$ Hermitian forms $\left\{\gamma_{i k}^{\beta}\right\}$ is positive definite.

We now apply our theory to the gauge function $f$ introduced by

$$
f^{2}=\operatorname{tr}\left(\gamma^{*}\left(\mathfrak{x}^{*}\right)\right) \text {. }
$$

In terms of the splits (17) one has

$$
f^{2}=\sum_{\alpha} g^{\alpha}+2 \sum_{\beta} g^{\beta} .
$$

The properties (i) to (iii) of $\S 4$ are readily verified; (ii*) is also fulfilled because of

$$
f^{2}\left(\tau^{*} \mathfrak{r}^{*}\right)=\sum_{\alpha}\left|\tau^{\alpha}\right|{ }^{2} g^{\alpha}+2 \sum_{\beta}\left|\tau^{\beta}\right|^{2} g^{\beta}
$$

6. Quaternion algebra of totally positive norm over a totally real field. Turning to noncommutative division algebras, we denote by 2 the quasifield of quaternions

$$
a=a_{0}+a_{1} i_{1}+a_{2} i_{2}+a_{3} i_{3}
$$

whose components $a_{0}, a_{1}, a_{2}, a_{3}$ are arbitrary real numbers, and use the notations $\bar{a}$ and $|a|$ in the customary manner: 


$$
a \bar{a}=|a|^{2}=a_{0}^{2}+a_{1}^{2}+a_{2}^{2}+a_{3}^{2} .
$$

For which of the noncommutative division algebras of finite degree over $R_{0}$ does the concept of infinite prime spots work in a way similar to that in the previous section for fields? I am going to describe one such situation without discussing the question whether or not it is the only one (though, as a matter of fact, it is).

Suppose we are given a field $\varepsilon$ of degree $e$ over $R_{0}$ and two numbers $\omega_{1}, \omega_{2}$ in $\varepsilon$. We put $\omega_{3}=\omega_{1} \omega_{2}$ and form the quaternion algebra $\mathcal{F}$ over $\mathcal{E}$ whose elements $\xi$ are quadruples $\left(\xi_{0}, \xi_{1}, \xi_{2}, \xi_{3}\right)$ of numbers in $\varepsilon$,

$$
\xi=\xi_{0}+\xi_{1 \iota_{1}}+\xi_{2 \iota_{2}}+\xi_{3} \iota_{3},
$$

with this multiplication table for the unities $\iota_{1}, \iota_{2}, \iota_{3}$ :

$$
\begin{gathered}
\iota_{1}^{2}=-\omega_{1}, \quad \iota_{2}^{2}=-\omega_{2}, \quad \iota_{3}^{2}=-\omega_{3} ; \\
\iota_{1} \iota_{2}=-\iota_{2} \iota_{1}=\iota_{3}, \quad \iota_{3} \iota_{1}=-\iota_{1} \iota_{3}=\omega_{1} \iota_{2}, \quad \iota_{2} \iota_{3}=-\iota_{3} \iota_{2}=\omega_{2} \iota_{1} .
\end{gathered}
$$

The conjugate $\bar{\xi}$ is $\xi_{0}-\xi_{1} \iota_{1}-\xi_{2} \iota_{2}-\xi_{3} \iota_{3}$ and

$$
\xi \bar{\xi}=\xi_{0}^{2}+\omega_{1} \xi_{1}^{2}+\omega_{2} \xi_{2}^{2}+\omega_{3} \xi_{3}^{2}
$$

If the equation

$$
\xi_{0}^{2}+\omega_{1} \xi_{1}^{2}+\omega_{2} \xi_{2}^{2}+\omega_{3} \xi_{3}^{2}=0
$$

has no solution $\left(\xi_{0}, \xi_{1}, \xi_{2}, \xi_{3}\right)$ in $\varepsilon$ except $(0,0,0,0)$, then $\mathcal{F}$ is a division algebra of degree 4 over $\varepsilon$ and of degree $f=4 e$ over $R_{0}$. We assume $\varepsilon$ to be totally real (to have no imaginary infinite prime spot) and $\omega_{1}, \omega_{2}$ to be totally positive numbers in $\varepsilon$ (i.e., cheir $e$ conjugates $\omega_{1}^{\alpha}, \omega_{2}^{\alpha}$ are all positive). Then the quadratic form of the variables $\xi_{0}, \xi_{1}, \xi_{2}, \xi_{3}$ at the left of (21) is positive definite in each conjugate $\mathcal{E}^{\alpha}$ of $\mathcal{E}$ and hence (21) has no solution except 0 . Denoting as before by $\tau^{\alpha}$ the conjugate of any number $\tau$ in $\varepsilon$ corresponding to the spot $\alpha$ of $\mathcal{E}$, we map (20) upon the element

$$
\xi^{\alpha}=\xi_{0}^{\alpha}+\xi_{1}^{\alpha}\left(\omega_{1}^{\alpha}\right)^{1 / 2} \cdot i_{1}+\xi_{2}^{\alpha}\left(\omega_{2}^{\alpha}\right)^{1 / 2} \cdot i_{2}+\xi_{3}^{\alpha}\left(\omega_{3}^{\alpha}\right)^{1 / 2} \cdot i_{3}
$$

in 2. This "conjugation" is an isomorphic mapping and defines the "infinite quaternion prime spot" $\alpha$ of $\mathcal{F}$. (22) are the splits, and the $4 e=f$ real numbers

$$
x_{0}^{\alpha}=\xi_{0}^{\alpha}, \quad x_{1}^{\alpha}=\xi_{1}^{\alpha}\left(\omega_{1}^{\alpha}\right)^{1 / 2}, \quad x_{2}^{\alpha}=\xi_{2}^{\alpha}\left(\omega_{2}^{\alpha}\right)^{1 / 2}, \quad x_{3}^{\alpha}=\xi_{3}^{\alpha}\left(\omega_{3}^{\alpha}\right)^{1 / 2}
$$

are the "splitting coordinates," of $\xi$. Application to the elements $\xi^{*}$, equation $\left(2^{*}\right)$, of $\mathcal{F}^{*}$ is immediate.

There is only one thing to settle: The splitting coordinates $x_{0}^{\alpha}, x_{1}^{\alpha}, x_{2}^{\alpha}, x_{3}^{\alpha}$ arise from the components $x_{a}$ by the substitution (11), each $\sigma_{a}$ standing for 
the column of its splitting coordinates. Is its determinant, whose absolute value will again be denoted by $\Delta$, different from zero? To answer the question, let $\left(\tau_{1}, \cdots, \tau_{e}\right)$ be a basis of $\varepsilon$ and set $\Delta_{0}=$ abs. $\left|\tau_{1}, \cdots, \tau_{e}\right|$. From it we obtain the following basis of $\mathcal{F}$ :

$$
\tau_{a}, \quad \tau_{a} \iota_{1}, \quad \tau_{a} \iota_{2}, \quad \tau_{a} \iota_{3} \quad(a=1, \cdots, e) .
$$

The $\Delta$ of this particular basis is given by

$$
\Delta=\prod_{\alpha}\left(\begin{array}{c}
\alpha \\
\omega_{1}^{\alpha} \omega_{2}^{\alpha}
\end{array}\right) \cdot \Delta_{0}^{4}=\operatorname{Nm} \omega_{3} \cdot \Delta_{0}^{4} .
$$

Thus $\Delta \neq 0$ for this and consequently for any basis.

Incidentally $\Delta$ is a rational number for any basis of $\mathcal{f}$ and $4^{\circ \cdot} \Delta$ a rational integer if $\sigma_{1}, \cdots, \sigma_{f}$ is a basis of [f]. The characteristic equation of the multiplication $\xi^{*} \rightarrow \xi^{*} \cdot \delta$ considered as a linear operation in $\mathcal{F}^{*}$ is the square of a polynomial (of degree $2 e$ ), and so is the characteristic polynomial of the linear substitution (4) in $E^{n f}$.

The notion of unit and the absolute value $\left|\xi^{*}\right|$ of any element $\xi^{*}$ of $\mathfrak{Z}^{*}$ are introduced as before. The constant on the right side of $\left(13_{k}\right)$ is to be changed into

$$
(k f) !\left(\frac{32}{\pi^{2}}\right)^{e k}\left(\frac{\Delta}{f !}\right)^{k}
$$

As gauge functions $f$ we employ in particular those whose square equals

$$
\frac{1}{4} \operatorname{tr}\left(\gamma^{*}\right)=\sum_{\alpha} g^{\alpha}
$$

where $\gamma^{*}$ is any positive Hermitian form (16) in $\mathcal{F}^{*}$.

7. The theorems of finiteness for quadratic forms. After so many preliminaries which stake out the ground covered by our investigation, I now come to the core of the matter, which may be explained fairly completely by the simplest example $\mathcal{F}=\mathcal{R}_{0}$. Here we have only one order $[\mathcal{f}]$ consisting of the ordinary integers $0, \pm 1, \pm 2, \cdots$ and only one class of lattices. For any given lattice $\Lambda$ over I and any positive quadratic form

$$
f^{2}(\mathfrak{x})=\sum g_{i k} x_{i} x_{k} \quad\left(g_{k i}=g_{i k}\right)
$$

the conditions of reduction read:

$$
f^{2}(\mathfrak{x}) \geqq g_{k k} \quad \text { whenever } \mathfrak{x}=\left(x_{1}, \cdots, x_{n}\right) \text { is in } \Lambda_{k} .
$$

Each of them is a linear inequality for the coefficients $g_{i j}$.

With the notation used in R1 we carry out Jacobi's transformation:

$$
f^{2}=q_{1} z_{1}^{2}+\cdots+q_{n} z_{n}^{2} .
$$

The volume $V$ of the ellipsoid $f^{2}<1$ is given by 


$$
V^{2}=\omega_{n}^{2} / q_{1} \cdots q_{n},
$$

$\omega_{n}$ being the volume of the $n$-dimensional sphere. Hence the inequality (12),

$$
M_{1} \cdots M_{n} V[\Lambda: \mathrm{I}] \leqq 2^{n},
$$

turns into

$$
g_{11} \cdots g_{n n}[\Lambda: I]^{2} \leqq\left(2^{n} / \omega_{n}\right)^{2} \cdot q_{1} \cdots q_{n} .
$$

As Minkowski observed, (23) may be proved much more easily for quadratic forms than for an arbitrary gauge function. By an argument similar to the one employed in proving Theorem II we see that the ellipsoid

$$
f^{\prime 2}=\frac{q_{1}}{M_{1}^{2}} z_{1}^{2}+\cdots+\frac{q_{n}}{M_{n}^{2}} z_{n}^{2}<1
$$

contains no lattice vector except zero. Hence its volume $V^{\prime}$ satisfies the inequality

$$
V^{\prime}[\Lambda: I] \leqq 2^{n}, \quad \text { and } V^{\prime}=M_{1} \cdots M_{n} \cdot V .
$$

If $\kappa_{n}$ is a number such that the part of space covered by impenetrable $n$-dimensional spheres in any lattice arrangement may never exceed the proportion $\kappa_{n}: 1$ then we can even write $\kappa_{n} 2^{n}$ instead of $2^{n}$ and thus replace $\omega_{n}$ in (24) by $\pi_{n}=\omega_{n} / \kappa_{n}$. The most primitive choice is $\kappa_{n}=1$; however, according to Blichfeldt's ingenious device $\left({ }^{6}\right)$,

$$
\kappa_{n}=(n+2) \cdot 2^{-1-n / 2}
$$

is a permissible and better value.

Making use of the inequalities

$$
g_{i i} \geqq q_{i}
$$

on the left side of (24) we get for the index $j=[\Lambda: I]$ this upper bound

$$
j \leqq 2^{n} / \pi_{n}
$$

which is a considerable improvement over (13), $j \leqq n$ ! For $n=1,2,3$ it yields the result $j=1$, to which the theory of reduction for binary and ternary forms owes its comparative simplicity.

For similar reasons

$$
\begin{aligned}
g_{11} \cdots g_{k k} \cdot j_{k}^{2} & \leqq\left(2^{k} / \pi_{k}\right)^{2} \cdot q_{1} \cdots q_{k}, \\
j_{k} & \leqq 2^{k} / \pi_{k}
\end{aligned} \quad(k=1, \cdots, n) .
$$

Unless the lattice $\Lambda$ satisfies the $n$ inequalities $\left(25_{k}\right)$ for its indices $j_{k}=\left[\Lambda^{(k)}: \mathrm{I}^{(k)}\right]$ there can be no $\Lambda$-reduced forms.

(8) H. F. Blichfeldt, Mathematische Annalen, vol. 101 (1929), pp. 605-608. 
Dividing $\left(24_{k}\right)$ by

$$
g_{11} \cdots g_{k-1, k-1} \geqq q_{1} \cdots q_{k-1}
$$

we find that our reduced form satisfies the fundamental relations

$$
q_{k} \geqq \lambda_{k} g_{k k}
$$

where

$$
\lambda_{k}=\left(j_{k} \pi_{k} / 2^{k}\right)^{2} .
$$

This lower bound for $q_{k}$ is much better than the corresponding one holding for the method of reduction studied in $\mathrm{R} 1$.

The first theorem of finiteness deals with the subset $\Lambda_{k}(=)$ of $\Lambda_{k}$ to which a vector $\mathfrak{r}$ in $\Lambda_{k}$ belongs if there exists a $\Lambda$-reduced positive quadratic form $f^{2}$ satisfying the equation $f^{2}(\mathfrak{x})=g_{k k}$. The set $\Lambda_{k}(=)$ is finite. The proof is as in $\mathrm{R} 1$, but the upper bounds arrived at are a good deal lower. The first part of the proof yields the bounds

$$
\lambda_{i} z_{i}^{2} \leqq 1 \quad(\text { for } i=k, k+1, \cdots, n)
$$

where the $\lambda_{i}$ are now defined by (27). In the second part one replaces the vector $\mathfrak{x}$ in $\Lambda_{k}(=)$ by $\mathfrak{x}-\mathfrak{x}_{h}$ where $\mathfrak{x}_{h}$ is any vector in $\Lambda^{(h)}(h<k)$ and observes that

$$
f^{2}\left(\mathfrak{x}-\mathfrak{x}_{h}\right) \geqq g_{k k} \text {. }
$$

This is true in particular if $\mathfrak{x}_{h}$ is in $\mathrm{I}^{(h)}$, and as in $\mathrm{R} 1$ one thus derives the relations

$$
\lambda_{h} z_{h}^{2} \leqq \rho^{2} h \quad(\rho=1 / 2 ; h=1, \cdots, k-1) .
$$

Once the discrete lattice $\Lambda$ is given, the resulting universal upper bounds for $\left|x_{n}\right|, \ldots,\left|x_{1}\right|$ leave only a limited number of possibilities for a vector $\mathfrak{x}=\left(x_{1}, \cdots, x_{n}\right)$ in $\Lambda$.

The second theorem of finiteness shall be restated in a more natural and slightly more general form. Let $p \geqq 1$ and $w \geqq 0$ be given. With respect to the lattice $\Lambda$ over I the positive quadratic form $f^{2}$ will be said to have the property $B(p, w)$ provided

$$
f^{2}(\mathfrak{x}) \geqq \frac{1}{p} \cdot f^{2}\left(\mathfrak{e}_{k}\right)
$$

for any vector $\mathfrak{x}$ in $\Lambda_{k}$, and

$$
f^{2}\left(\mathfrak{e}_{k}-\mathfrak{x}_{h}\right) \geqq f^{2}\left(\mathfrak{e}_{k}\right)-w \cdot f^{2}\left(\mathfrak{e}_{h}\right)
$$

for $h<k$ and any vector $\mathfrak{x}_{h}$ in $\Lambda^{(h)}$. Again, each of these conditions is a linear inequality for the coefficients $g_{i j}$ of $f^{2}$. We maintain:

Given two lattices $\Lambda$ and $\Lambda^{\prime}$ over $\mathrm{I}$, there is only a limited number of linear 
transformations carrying $\Lambda$ into $\Lambda^{\prime}$ and at the same time capable of carrying an unspecified $\Lambda$-reduced form $f^{2}$ into an unspecified $f^{\prime 2}$ which has the property $B(p, w)$ with respect to $\Lambda^{\prime}$.

We write the transformation as

$$
\mathfrak{x}=\sum_{i} x_{i} e_{i}=\sum_{i} y_{i} \grave{\jmath}_{i}:
$$

if $\left(x_{1}, \cdots, x_{n}\right)$ is in $\Lambda$, then $\left(y_{1}, \cdots, y_{n}\right)$ is in $\Lambda^{\prime}$, and vice versa. In particular, the $\delta_{1}, \cdots, \delta_{n}$ are vectors in $\Lambda .\left(p, e_{i}, \delta_{i}\right.$ were denoted in R1 by $\rho^{2}, \mathfrak{b}_{i}, \mathfrak{B}_{i}$. $)$ More explicitly as has been done in $\mathrm{R} 1$, we divide the row of indices $1, \cdots, n$ into a number of sections by means of the subspaces

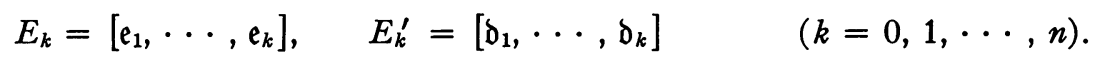

We pick out those $k=l_{0}, l_{1}, \cdots, l_{v}$,

$$
0=l_{0}<l_{1}<\cdots<l_{v-1}<l_{v}=n
$$

for which $E_{k}=E_{k}^{\prime}$, and divide the range of $k$ into the $v$ sections

$$
l_{u-1}<k \leqq l_{u} \quad(u=1, \cdots, v) .
$$

We then study the possibilities for transformations $\left(\delta_{1}, \cdots, \delta_{n}\right)$ with given $l_{1}, \cdots, l_{v-1}$.

By the analogues of Theorems $8_{p}$ and $9_{p}$ we have

$$
g_{k k}^{\prime} \leqq p g_{k k}
$$$$
(k=1, \cdots, n)
$$

and moreover

$$
g_{i+1, i+1} \leqq p g_{i i}
$$

whenever $i$ and $i+1$ are in the same section. Consider a $\delta_{k}$ of the last section $\left(l_{v-1}<k \leqq n\right)$. The first part of the proof in R1 yields for $\mathfrak{x}=\delta_{k}$ the simple upper bounds

$$
\lambda_{h} z_{h}^{2} \leqq p^{\{k-h\}+1}
$$

if $h$ also belongs to the last section, $\{k\}$ denoting 0 or $k$ according as $k \leqq 0$ or $k>0$. The second part requires a slight modification. Suppose $h$ lies in the $u$ th section $(u<v)$, and set for the moment $l_{u}=l$. Since $E_{l}=E_{l}^{\prime}$, the vectors in $\Lambda^{(l)}$ are obtained from the expression $y_{1} b_{1}+\cdots+y_{l} \delta_{l}$ by running $\left(y_{1}, \cdots, y_{l}\right.$, $0, \cdots, 0)$ over $\Lambda^{\prime(l)}$. Hence, according to the postulate $\left(28^{\prime}\right)$ :

$$
f^{\prime 2}\left(\mathfrak{e}_{k}-\mathfrak{y}^{\prime}\right) \geqq g_{k k}^{\prime}-w g_{l l}^{\prime}
$$

for any vector $\mathfrak{y}^{\prime}$ in $\Lambda^{\prime(l)}$, or

$$
f^{2}\left(\mathfrak{d}_{k}-\mathfrak{x}^{\prime}\right) \geqq g_{k k}^{\prime}-w g_{l l}^{\prime}
$$


for any vector $\mathfrak{x}^{\prime}$ in $\Lambda^{(l)}$, a fortiori for any vector in $\Lambda^{(h)}$, a fortior $i$ for any vector $\mathfrak{x}^{\prime}$ in $\mathbf{I}^{(h)}$. Following the same argument as in $\mathrm{R} 1$, one gets the inequality

$$
w g_{l l}^{\prime}+\rho^{2} h g_{h h} \geqq \lambda_{h} g_{h h} z_{h}^{2}
$$$$
(\rho=1 / 2) .
$$

But because $h$ and $l$ are in the same section, (30) and (29) lead to

$$
g_{l l} \leqq p^{l-h} g_{h h}, \quad g_{l l}^{\prime} \leqq p g_{l l} \leqq p^{l-h+1} g_{h h}
$$

and thus finally

$$
\lambda_{h} z_{h}^{2} \leqq h \rho^{2}+w \cdot p^{l_{u-h+1}} \quad\left(l_{u-1}<h \leqq l_{u} ; u=1, \cdots, v-1\right) .
$$

It is clear how the same procedure applies to a $k$ in the lower sections. De. noting the values of the variables $z_{1}, \cdots, z_{n}$ for $\mathfrak{x}=\mathfrak{d}_{k}$ by $z_{1 k}, \cdots, z_{n k}$, one finds:

$z_{h k}=0$ if $h$ is in a higher section than $k$;

$\lambda_{h} z_{h k}^{2} \leqq p^{\{k-h\}+1}$ if $h$ and $k$ are in the same section;

$\lambda_{h} z_{h k}^{2} \leqq h \rho^{2}+w \cdot p^{l-h+1}$ if $h$ is in a lower section than $k$ which ends with $l$.

8. Modifications in arbitrary fields and quasi-fields. Our next concern is to examine whether any serious modifications of the procedure just described arise in the two general cases of a field and a quaternion algebra over a field. Take the case of the field first. With a positive Hermitian form $\gamma^{*}$ in $\mathcal{F}^{*}$ we combine its trace $f^{2}$ :

$$
f^{2}\left(\xi_{1}^{*}, \cdots, \xi_{n}^{*}\right)=\sum_{\alpha} \sum_{i, k} g_{i k}^{\alpha} x_{i}^{\alpha} x_{k}^{\alpha}+2 \sum_{\beta} \sum_{i, k} \gamma_{i k}^{\beta} \xi_{i}^{\beta} \bar{\xi}_{k}^{\beta} \quad\left(\xi_{k}^{\beta}=x_{k 0}^{\beta}+i x_{k 1}^{\beta}\right) .
$$

$\gamma^{*}$ is called reduced with respect to $\Lambda$ if the gauge function $f$ is, i.e., if

$$
f^{2}\left(\xi_{1}, \cdots, \xi_{n}\right) \geqq \operatorname{tr}\left(\gamma_{k k}^{*}\right)=M_{k}^{2}
$$

for any vector $\left(\xi_{1}, \cdots, \xi_{n}\right)$ in $\Lambda_{k}$. Each part is subjected to its Jacobi transformation:

Besides

$$
\begin{aligned}
& \sum_{i, k} g_{i k}^{\alpha} x_{i}^{\alpha} x_{k}^{\alpha}=\sum_{i} q_{i}^{\alpha}\left(z_{i}^{\alpha}\right)^{2}, \\
& \sum_{i, k} \gamma_{i k}^{\beta} \xi_{i}^{\beta} \bar{\xi}_{k}^{\beta}=\sum_{i} q_{i}^{\beta}\left|\xi_{i}^{\beta}\right|^{2} .
\end{aligned}
$$

$$
\operatorname{tr}\left(q_{i}\right)=\sum_{\alpha} q_{i}^{\alpha}+2 \sum_{\beta} q_{i}^{\beta}, \quad \operatorname{Nm}\left(q_{i}\right)=\prod_{\alpha} q_{i}^{\alpha} \cdot \prod_{\beta}\left(q_{i}^{\beta}\right)^{2}
$$

we introduce the mean value $\left\langle q_{i}\right\rangle$ by

$$
f \cdot\left\langle q_{i}\right\rangle=\operatorname{tr}\left(q_{i}\right) .
$$


In terms of the coordinates $x_{k}^{\alpha} ; x_{\mathbf{k} 0}^{\beta}, x_{\mathbf{k} 1}^{\beta}$ the volume $V$ of the ellipsoid $f^{2}\left(\mathfrak{r}^{*}\right)<1$ is

$$
\omega_{n f} \text { divided by } 2^{n s}\left(\prod_{i} \mathrm{Nm} q_{i}\right)^{1 / 2} \text {. }
$$

Instead of applying Minkowski's second inequality to the present gauge function we again consider the ellipsoid

$$
f^{\prime 2}\left(\mathfrak{x}^{*}\right)=\operatorname{tr}\left(\sum_{i} \frac{q_{i}}{M_{i}^{2}} \zeta_{i} \bar{\zeta}_{i}\right)<1
$$

which contains no lattice vector except zero, and thus establish the inequality

$$
\prod_{i}\left(\operatorname{tr} \gamma_{i i}^{*}\right)^{f} \cdot[\Lambda: \mathrm{I}]^{2} \leqq \frac{\left(4^{r+s} \Delta^{2}\right)^{n}}{\pi^{2} n_{f}} \cdot \prod_{i} \operatorname{Nm} q_{i}
$$

for any reduced $\gamma^{*} / \Lambda$.

Now enters the only new feature: Making use of the inequality between arithmetic and geometric means in the form

$$
\operatorname{Nm} q_{i} \leqq\left\langle q_{i}\right\rangle^{\gamma}
$$

we infer from

$$
\stackrel{\alpha}{q_{i}} \leqq \stackrel{\alpha}{g_{i i}}, \quad \underline{q}_{i}^{\beta} \leqq \stackrel{\beta}{\gamma_{i i}}
$$

the relation

$$
\left\langle\gamma_{i i}^{*}\right\rangle^{\gamma} \geqq\left\langle q_{i}\right\rangle^{f} \geqq \mathrm{Nm} q_{i},
$$

and then (33) yields the following upper bound for $j=[\Lambda: I]$ :

$$
j \leqq 1 / \mu_{n} \text { with the abbreviation } \mu_{n}=\frac{\pi_{n f} \cdot f^{n f / 2}}{\left(2^{r+s} \Delta\right)^{n}} .
$$

For the same reasons

$$
\prod_{i=1}^{k}\left\langle\gamma_{i i}^{*}\right\rangle^{\prime}\left(\mu_{k} j_{k}\right)^{2} \leqq \prod_{i=1}^{k} \mathrm{Nm}_{i} q_{i}
$$

and hence

$$
\mu_{k} j_{k} \leqq 1 .
$$

These estimates are an improved substitute for Theorem II. Combining (34) with

$$
\left.\prod_{i=1}^{k-1}\left\langle\gamma_{i i}^{*}\right\rangle\right\rangle^{f} \geqq \prod_{i=1}^{k-1}\left\langle q_{i}\right\rangle^{f} \geqq \prod_{i=1}^{k-1} \mathrm{Nm} q_{i}
$$


one gets

$$
\operatorname{Nm} q_{k} \geqq\left(\mu_{k} j_{k}\right)^{2} \cdot\left\langle\gamma_{k k}^{*}\right\rangle^{\gamma}
$$

Not only does this inequality establish a lower bound for the trace of $q_{k}$,

$$
\operatorname{tr} q_{k} \geqq\left(\mu_{k} j_{k}\right)^{2 / s} \cdot \operatorname{tr} \gamma_{k k}^{*},
$$

but it also shows that the geometric mean of the conjugates of $q_{k}$ is not much smaller than their arithmetic mean. Therefore none of the conjugates can be much smaller than their arithmetic mean. We have a special case of the situation dealt with by the following

Lemma 5. Let $f_{1}, \cdots, f_{m}$ be positive integers and $u_{1}, \cdots, u_{m} ; v_{1}, \cdots, v_{m}$ two rows of positive numbers. We set

$$
\begin{aligned}
f & =f_{1}+\cdots+f_{m}, \\
f \cdot\langle u\rangle & =f_{1} u_{1}+\cdots+f_{m} u_{m}, \\
\operatorname{Nm} u & =u_{1}^{f_{1}} \cdots u_{m}^{f_{m}} .
\end{aligned}
$$

If $u_{\alpha} \leqq v_{\alpha}(\alpha=1, \cdots, m)$ and

$$
\operatorname{Nm} u \geqq \mu \cdot\langle v\rangle^{f}
$$

with some constant $\mu \geqq 1$, then

$$
u_{\alpha} \geqq \lambda_{\alpha} \cdot\langle v\rangle
$$

where $\lambda_{\alpha}$ depends on $\mu$ but not on the $u$ and $v$.

(In our case $r$ among the weights $f_{\alpha}$ are 1 and $s$ of them equal 2.)

Proof. In the trivial case $m=1$ one determines $\lambda$ by

$$
\lambda^{f}=\mu \text {. }
$$

If $m>1$ we set $u_{1}=\lambda \cdot\langle v\rangle$ and assume $\lambda \leqq 1$. Then

$$
\operatorname{Nm} u=\lambda^{f_{1}}\langle v\rangle^{f_{1}} \cdot u_{2}^{f_{2}} \cdots u_{m}^{f_{m}} \leqq \lambda^{f_{1}}\langle v\rangle^{f_{1}}\langle u\rangle_{1}^{f-f_{1}}
$$

Here $\langle u\rangle_{1}$ denotes the arithmetic mean of $u_{2}, \cdots, u_{m}$ formed with the weights $f_{2}, \cdots, f_{m}$ of sum $f-f_{1}$ :

$$
\begin{aligned}
\left(f-f_{1}\right) \cdot\langle u\rangle_{1} & =f_{2} u_{2}+\cdots+f_{m} u_{m}=f \cdot\langle u\rangle-f_{1} u_{1} \\
& =f \cdot\langle u\rangle-f_{1} \lambda\langle v\rangle \leqq\left(f-f_{1} \lambda\right)\langle v\rangle .
\end{aligned}
$$

Therefore

$$
\operatorname{Nm} u \leqq \mu \cdot\langle v\rangle^{f}
$$

where 


$$
\mu=\lambda^{f_{1}}\left(\frac{f-f_{1} \lambda}{f-f_{1}}\right)^{f-f_{1}}
$$

As its logarithmic derivative shows,

$$
\frac{d \mu}{\mu}=\frac{f_{1}(1-\lambda)}{f-f_{1} \lambda} \cdot \frac{f d \lambda}{\lambda}
$$

this function $\mu(\lambda)$ maps the interval $0 \leqq \lambda \leqq 1$ monotonically upon $0 \leqq \mu \leqq 1$ and thus will assume the given value $\mu(\leqq 1)$ for a certain $\lambda=\lambda_{1}(\leqq 1)$. Thus we cannot have $u_{1}\left\langle\lambda_{1} \cdot\langle v\rangle\right.$ under the condition (37).

(We wish to obtain the best value for the constant $\lambda_{1}$. If one is content with a little less, one may choose $\lambda_{1}$ according to the equation

$$
\lambda_{1}^{f_{1}}\left(\frac{f}{f-f_{1}}\right)^{f-f_{1}}=\mu
$$

or even, as

$$
\begin{aligned}
\left(1+\frac{f_{1}}{f-f_{1}}\right)^{\left(f-f_{1} / f_{1}\right.}<\mathrm{e} \quad(=\text { basis of natural logarithms) }, \\
\left(\lambda_{1} \mathrm{e}\right)^{f_{1}}=\mu .
\end{aligned}
$$

Incidentally, formula (40) holds good also for the function (38) which rules the trivial case $m=1, f_{1}=f$.)

In this way we ascertain constants $\lambda_{k}, \lambda_{k}^{\prime}$ such that

$$
\text { each } q_{k}^{\alpha} \geqq \lambda_{k} \cdot\left\langle{ }^{*}{ }_{k k}\right\rangle \text { and each } q_{k}^{\beta} \geqq \lambda_{k}^{\prime} \cdot\left\langle\gamma_{k k}^{*}\right\rangle .
$$

In case there is only one infinite prime spot, $\lambda_{k}$ and $\lambda_{k}{ }^{\prime}$ are determined by the relation

$$
\left(\mu_{k} j_{k}\right)^{2}=\lambda_{k}=\lambda_{k}^{\prime 2}
$$

in case of several spots by the equations

$$
\left(\mu_{k} j_{k}\right)^{2}=\lambda_{k}\left(\frac{f-\lambda_{k}}{f-1}\right)^{f-1}=\lambda_{k}^{\prime 2}\left(\frac{f-2 \lambda_{k}^{\prime}}{f-2}\right)^{f-2}
$$

together with $\lambda_{k} \leqq 1$ and $\lambda_{k}^{\prime} \leqq 1$.

Similarly for the other case studied in $\S 6$, that of a quaternion algebra $\mathcal{F}$ with totally positive relative norm over a totally real field. The constants $\mu_{k}, \lambda_{k}$ in the inequalities

$$
\mu_{k} j_{k} \leqq 1 \text { and } q_{k}^{\alpha} \geqq \lambda_{k} \cdot\left\langle\gamma_{k k}^{*}\right\rangle
$$

are then given by

$$
\mu_{k}=\pi_{4 k e} \Delta^{-k}(e / 4)^{2 k e},
$$




$$
\left(\mu_{k} j_{k}\right)^{1 / 2}=\lambda_{k} \quad \text { or } \quad \lambda_{k}\left(\frac{e-\lambda_{k}}{e-1}\right)^{e-1} \quad\left(\lambda_{k} \leqq 1\right),
$$

according as $e=\frac{1}{4} f$ is 1 or is greater than 1 .

After this the proofs for the first and second theorems of finiteness roll along as before.

9. The pattern of equivalent cells. The Hermitian forms $\left\{\gamma_{i k}^{*}\right\}$ constitute a linear space of

$$
N=f \cdot \frac{1}{2} n(n-1)+(r+s) n=f \cdot \frac{1}{2} n(n+1)-s n
$$

or

$$
N=e n(2 n-1)
$$

(quasi-field $\mathcal{7}$ )

dimensions, the positive ones a convex cone $G$ in that space. $G$ is an open set; we operate within $G$ throughout. "Form" means any positive Hermitian form.

Let $\Lambda$ be a lattice over I. A $\Lambda$-reduced form $\gamma^{*}$ has been characterized by the inequalities

$$
f^{2}\left(\xi_{1}, \cdots, \xi_{n}\right) \geqq f^{2}\left(e_{1 k}, \cdots, e_{n k}\right)
$$

holding for $f^{2}=\operatorname{tr}\left(\gamma^{*}\right)$ whenever $\left(\xi_{1}, \cdots, \xi_{n}\right)$ is in $\Lambda_{k}$. For a given vector $\left(\xi_{1}, \cdots, \xi_{n}\right)$ the equality sign in (32) will hold identically for all Hermitian forms $\gamma^{*}$ only if

$$
\left(\xi_{1}, \cdots, \xi_{n}\right)=\epsilon \cdot\left(e_{1 k}, \cdots, e_{n k}\right) \quad(\epsilon \text { a unit }),
$$

as follows at once from the expression (31). For any other vector $\left(\xi_{1}, \cdots, \xi_{n}\right)$ the equation determines a $(N-1)$-dimensional hyperplane in our $N$-dimensional linear space of forms. This remark justifies our definition of "properly reduced" in terms of the group $U$ of units.

The forms $\gamma^{*}$ which are reduced with respect to $\Lambda$ make up a convex cone $G_{\Lambda}$ in $G$. The properly reduced forms are the inner points of $G_{\Lambda}$; see R1, p. 150 . $G_{\Lambda}$ may be empty; indeed it will be so unless the indices $j_{k}$ of $\Lambda$ satisfy the inequalities (35). Even if it is not empty it may be without inner points. Theorem 10 in R1, together with the first theorem of finiteness, proves:

THEOREM IV. If $G_{\Lambda}$ has inner points, then $G_{\Lambda}$ is a convex pyramid defined within $G$ by a limited number of linear inequalities.

A linear mapping $\mathfrak{x} \rightarrow \mathfrak{x}^{\prime}$ of $E^{n} / \mathcal{F}$ upon itself is one satisfying the conditions $\left(\mathfrak{x}_{1}+\mathfrak{x}_{2}\right)^{\prime}=\mathfrak{x}_{1}^{\prime}+\mathfrak{x}^{\prime}{ }_{2}$ and $(\delta \cdot \mathfrak{x})^{\prime}=\delta \cdot \mathfrak{x}^{\prime}$ for any number $\delta$ in $\mathcal{F}$. We also require that $\mathfrak{r}=0$ is the only vector whose image $\mathfrak{x}^{\prime}$ equals 0 . If $\delta_{1}, \cdots, \mathfrak{D}_{n}$ is any basis of $E^{n} / \mathcal{F}$ the mapping $S$ may be defined by giving the images $\delta_{i}^{\prime}=\grave{D}_{i} S$ of the $\delta_{2}$. The mapping $S$ carries a form $\gamma^{*}$ into a form $\gamma_{S}^{*}$ according to the equation $\gamma_{S}^{*}(\mathfrak{x} S)=\gamma^{*}(\mathfrak{x})$. An order [f] in $\mathcal{F}$ and a lattice $\mathfrak{R}$ belonging to the order [f] are supposed to be given. The linear mappings $S$ which leave $\mathfrak{R}$ invariant are 
said to form the modular group $\left({ }^{7}\right)$. In terms of a basis $\delta_{1}, \cdots, \delta_{n}$ of $E^{n} / \mathcal{F}$ the lattice $\mathfrak{R}$ is represented by $\Lambda$ :

$$
\mathfrak{x}=\eta_{1} \delta_{1}+\cdots+\eta_{n} \delta_{n} \quad \text { in } \quad \mathcal{L} \cdot \rightleftarrows \cdot\left(\eta_{1}, \cdots, \eta_{n}\right) \text { in } \Lambda,
$$

and a form $\gamma^{*}(\mathfrak{x})$ is represented by a form $\Gamma^{*}\left(\eta_{1}, \cdots, \eta_{n}\right)$ :

$$
\gamma^{*}\left(\eta_{1} \delta_{1}+\cdots+\eta_{n} \delta_{n}\right)=\Gamma^{*}\left(\eta_{1}, \cdots, \eta_{n}\right) .
$$

The linear mapping defined by $\delta_{i} \rightarrow \delta_{i}^{\prime}$ carries $\eta_{1} \delta_{1}+\cdots+\eta_{n} \delta_{n}$ into $\eta_{1} \delta_{1}^{\prime}+\cdots+\eta_{n} \delta_{n}^{\prime}$; hence it leaves $\mathcal{R}$ invariant and thus belongs to the modular group if and only if $\Lambda=\Lambda^{\prime}, \Lambda$ and $\Lambda^{\prime}$ being the representations of $\mathfrak{R}$ in terms of $\boldsymbol{b}_{i}$ and $\boldsymbol{b}_{i}^{\prime}$. For a vector $\mathfrak{b}$ in $\mathfrak{l}$ there are not more than a finite number of units $\epsilon$ such that $\epsilon \mathfrak{D}$ also is in $\mathfrak{R}$. Indeed, for the splits of $\mathfrak{x}=\epsilon \delta$ one finds

$$
\left|\xi^{\alpha}\right|=\left|\delta^{\alpha}\right|,\left|\xi^{\beta}\right|=\left|\delta^{\beta}\right|, \text { a fortiori }\left|\xi^{\alpha}\right| \leqq\left|\delta^{\alpha}\right|,\left|\xi^{\beta}\right| \leqq\left|\delta^{\beta}\right|,
$$

which in view of the discrete nature of $\mathfrak{R}$ proves the point.

We want to divide $G$ without gaps and overlappings into domains which are mutually equivalent under the modular group. We shall introduce these cells first as entities which have nothing to do with Hermitian forms, adopting a criterion of identity other than the set-theoretic one. The systematic place for this introduction would have been at the end of \$3. Only afterwards shall we explain the meaning of the phrase "a form lies in a cell." Here are the definitions:

A semi-basis $\delta_{1}, \cdots, \delta_{n}$ of $\mathfrak{R}$ determines a cell $Z\left(\mathfrak{b}_{1}, \cdots, \delta_{n}\right)$; the semibasis $\mathfrak{b}_{1}, \cdots, \mathfrak{b}_{n}$ is said to determine the same cell if

$$
\mathfrak{b}_{i}=\epsilon_{i} \widehat{\delta}_{i} \quad \text { ( } \epsilon_{i} \text { units). }
$$

Let $S$ be an operation of the modular group. The image $Z_{S}$ of $Z=Z\left(\delta_{1}, \cdots, \delta_{n}\right)$ is defined as $Z\left(\delta_{1}^{\prime}, \cdots, \mathfrak{b}_{n}^{\prime}\right)$ where $\mathfrak{b}_{i}^{\prime}=\grave{b}_{i} S$. (Notice that if $Z$ is written as $Z\left(\mathfrak{b}_{1}, \cdots, \mathfrak{b}_{n}\right), \mathfrak{b}_{i}=\epsilon_{i} \mathfrak{b}_{i}$, then $Z\left(\mathfrak{b}_{1}^{\prime}, \cdots, \mathfrak{b}_{n}^{\prime}\right)$ is the same $Z_{S}$ because $\mathfrak{b}_{i}^{\prime}=\epsilon_{i} \mathfrak{b}_{i}^{\prime}$; thus $Z_{S}$ is independent of the fixation of the defining semi-basis $\grave{D}_{1}, \cdots, \mathfrak{b}_{n}$.) Those $S$ of the modular group for which $Z_{S}=Z$ shall be denoted by $J_{Z}$; they form a finite group $\left\{J_{Z}\right\}$. Indeed, for such an $S=J_{Z}$ one must have

$$
\mathfrak{d}_{i}^{\prime}=\mathfrak{d}_{i} S=\sigma_{i} \grave{D}_{i}
$$

( $\sigma_{i}$ a unit),

and the $J_{Z}$ are those mappings of the special form (43) which leave $\mathfrak{R}$ invariant. (In terms of another defining semi-basis $\mathfrak{b}_{i}=\epsilon_{i} \mathfrak{d}_{i}$ the same $J_{Z}$ is expressed by $\mathfrak{b}_{i}^{\prime}=\epsilon_{i} \sigma_{i} \epsilon_{i}^{-1} \cdot \mathfrak{b}_{i}$.) Any operation $S$ of the modular group has the same effect upon $Z$ as $J_{Z} S$.

$\left.{ }^{7}\right)$ If one feels that this term ought to be reserved for the group which is fundamental in the theory of the modules of the theta functions then a new word, say "lattice group," is indicated for our purpose. 
In terms of $\left(b_{1}, \cdots, b_{n}\right)$ the lattice $\mathfrak{R}$ is represented by an admissible lattice $\Lambda$, i.e., by a lattice $\Lambda$ over I which is equivalent to $R$. Hence to the cell $Z=Z\left(\mathfrak{b}_{1}, \cdots, \delta_{n}\right)$ there corresponds a family of admissible lattices $\Lambda$, and the same family to each equivalent cell $Z_{S}$. We have a one-to-one correspondence between the classes of equivalent cells on the one hand, and the families of admissible lattices $\Lambda$ on the other. We distinguish them by different colors. The operations $J_{Z}$ are represented by the operations $J_{\Lambda}$ in terms of the basis $\left(\delta_{1}, \cdots, \delta_{n}\right)$.

Now we come to the realization of cells as point sets in $G$. A form $\gamma^{*}$ is said to lie in $Z=Z\left(\delta_{1}, \cdots, \delta_{n}\right)$ if $\left(\delta_{1}, \cdots, \delta_{n}\right)$ is reduced with respect to $\gamma^{*}$, i.e., if for $f^{2}=\operatorname{tr}\left(\gamma^{*}\right)$ one has $f^{2}(\mathfrak{x}) \geqq f^{2}\left(\mathfrak{b}_{k}\right)$ whenever $\mathfrak{x}$ is in $\mathfrak{R}$ and outside $\left[b_{1}, \cdots, \delta_{k-1}\right]$. Because (42) implies

$$
f^{2}\left(\mathfrak{b}_{k}\right)=f^{2}\left(\mathfrak{b}_{k}\right), \quad\left[\mathfrak{b}_{1}, \cdots, \mathfrak{b}_{k-1}\right]=\left[\mathfrak{b}_{1}, \cdots, \mathfrak{b}_{k-1}\right],
$$

the definition is independent of the fixation of the defining semi-basis

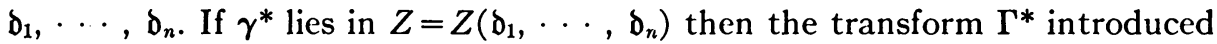
by (41) lies in $G_{\Lambda}$, and $\gamma_{S}{ }^{*}$ lies in $Z_{S}$.

The fact that there always exists a reduced semi-basis for a given $\gamma^{*}$ and the concluding sentence of $\$ 3$ can now be stated thus:

(a) Every point $\gamma^{*}$ lies in at least one cell $Z$.

(b) An inner point of a cell $Z$ cannot lie in a cell $Z^{\prime}$ unless $Z^{\prime}$ is the same as $Z$ (or briefly: different cells have no inner points in common).

The fact (a) will of course not be altered by suppressing all empty cells and their colors. Thus we have to look only for those admissible $\Lambda$ whose indices satisfy the conditions (35); and this brings the colors down to a limited number. Will (a) still prevail after suppressing all cells without inner points and their colors? The answer is affirmative because there is no inner clustering of cells in $G$. This is a consequence of the second theorem of finiteness, which now takes on the following form. Let $\mathfrak{a}_{1}, \cdots, \mathfrak{a}_{n}$ be a semi-basis of $\mathfrak{R}, p \geqq 1$ and $w \geqq 0$. The form $\gamma^{*}$ is said to lie in $Z\left(a_{1}, \cdots, a_{n} \mid p, w\right)$ if

$$
f^{2}(\mathfrak{x}) \geqq \frac{1}{p} \cdot f^{2}\left(\mathfrak{a}_{k}\right)
$$

whenever $\mathfrak{x}$ is in $\mathfrak{R}$ and outside $\left[\mathfrak{a}_{1}, \cdots, \mathfrak{a}_{k-1}\right]$, and if, moreover,

$$
f^{2}\left(\mathfrak{a}_{k}-\mathfrak{x}_{h}\right) \geqq f^{2}\left(\mathfrak{a}_{k}\right)-w \cdot f^{2}\left(\mathfrak{a}_{h}\right)
$$

whenever $h<k$ and $\mathfrak{x}_{h}$ is in $\mathfrak{R}$ and $\left[\mathfrak{a}_{1}, \cdots, \mathfrak{a}_{h}\right]$.

THEOREM V. There is only a finite number of operations $S$ of the modular group such that the image $Z_{S}$ into which a given cell $Z$ is thrown by $S$ will have points in common with the domain $Z\left(\mathfrak{a}_{1}, \cdots, \mathfrak{a}_{n} \mid p, w\right)$.

Application to $p=1, w=0$ proves in particular that a cell borders on not more than a finite number of other cells. And since $Z\left(\mathfrak{a}_{1}, \cdots, \mathfrak{a}_{n} \mid p, w\right)$ sweeps 
over the whole $G$ if $p$ and $w$ increase to infinity we are sure that the cells cluster around no point in the interior of $G$ ("the modular group is properly discontinuous in $G$ "). We therefore definitely admit only those colors whose cells are $N$-dimensional solids, i.e., have inner points. In our summary we talk of them as point sets in $G$.

Theorem VI. (a) $G$ is divided into a pattern of cells, each cell bearing a color out of a finite palette of colors. The cells cover $G$ without gaps and overlappings. Each cell is a solid convex pyramid (in $G$ ). The mappings of the modular group leave this design, including its coloring, invariant. Any two cells of the same color can be carried one into the other by an operation of the modular group.

(b) Given a point in $G$ and a cell $Z$ one can assign a neighborhood $\mathfrak{N}$ to the point such that there is only a limited number of operations $S$ of the modular group for which the image $Z_{S}$ penetrates into $\mathfrak{N}$.

(c) The operations of the modular group which carry a cell into itself form a finite subgroup. This group of linear operations in the vector space $E^{n} / \mathcal{F}$ is equivalent (in F) to a group whose elements are of the special form

$$
\xi_{1} \rightarrow \xi_{1} \epsilon_{1}, \cdots, \xi_{n} \rightarrow \xi_{n} \epsilon_{n}
$$

( $\epsilon_{i}$ units).

(Of course, in view of statement (c)' the statement (b) could have been replaced by the simpler one that only a finite number of cells penetrate into $\mathfrak{N}$.)

We form a nucleus by selecting one cell $Z_{c}$ of each color $c$. All cells adjacent to the nuclear cells form a wreath around the nucleus. Here the word "adjacent" may be interpreted either in the wide sense of "having a point in common," or in the narrower sense of "having a wall of $N-1$ dimensions in common."

Theorem VII. Determine for each cell $Z_{c}^{\prime}$ of color $c$ in the wreath an operation $S_{c}^{\prime}$ of the modular group which maps the nuclear cell $Z_{c}$ of color $c$ into $Z_{c}^{\prime}$. The $S_{c}^{\prime}$ thus selected, together with the operations $J_{c}$ of the modular group which carry $Z_{c}$ into itself, generate the whole group if all colors $c$ are taken into account.

Were it not for the groups $\left\{J_{\Lambda}\right\}$ the nucleus would form a fundamental domain. As it is, one has first to replace in our construction each $G_{\Lambda}$ by a part $G_{\Lambda}{ }^{\prime}$ which in $G_{\Lambda}$ is a fundamental domain for the finite group of special transformations

$$
J_{\Lambda}: \quad \xi_{k} \rightarrow \xi_{k} \epsilon_{k}
$$

carrying $G_{\Lambda}$ into itself. The effect of $J_{\Lambda}$ upon the coefficient $\gamma_{i k}^{*}$ is described by

$$
\stackrel{\gamma_{i k}^{*}}{\rightarrow} \epsilon_{i} \gamma_{i k}^{*} \bar{\epsilon}_{k} \text {. }
$$

If in one split $\alpha$ the transformation of the variable $\gamma_{i k}^{\alpha}=\Xi$,

$$
\left.\Xi \rightarrow \underset{\epsilon_{i}}{\alpha} \Xi_{\epsilon}^{-\alpha} \epsilon_{k}^{\alpha}=\underset{\epsilon_{i}}{\alpha} \Xi_{\left(\epsilon_{k}\right.}^{\alpha}\right)^{-1},
$$


is the identity, then the same is true of every split. Hence it is sufficient to consider one split $\alpha$ only, and after choosing it we write simply $\gamma_{i k}^{\alpha}=\gamma_{i k}$, $\epsilon_{i}^{\alpha}=\epsilon_{i}$. If the transformation $\Xi \rightarrow \epsilon_{1} \Xi_{1} \epsilon_{2}^{-1}$ is the identity, one must have $\epsilon_{1}=\epsilon_{2}$ as the specialization $\Xi=1$ shows, and $\Xi \rightarrow \epsilon_{2} \Xi \epsilon_{1}^{-1}$ is also the identity. Moreover, if $\Xi \rightarrow \epsilon_{1} \Xi \epsilon_{2}^{-1}$ and $\Xi \rightarrow \epsilon_{2} \Xi \epsilon_{3}^{-1}$ are identities, then $\Xi \rightarrow \epsilon_{1} \Xi_{\epsilon_{3}}^{-1}$ is. Consequently we may well limit ourselves first to the coefficients $\gamma_{i k}(i<k)$ on one side of the diagonal, and then more particularly to

$$
\Xi_{1}=\gamma_{12}, \Xi_{2}=\gamma_{23}, \cdots, \Xi_{n-1}=\gamma_{n-1, n} .
$$

Let us at once consider the most disagreeable case, that of a quaternion quasifield $\mathcal{F}$ as described in $\S 6$.

The group $\left\{J_{\Lambda}\right\}$ induces a group of transformations of the type

$$
\Xi \rightarrow \epsilon_{1} \Xi \overline{\epsilon_{2}} \quad\left(\left|\epsilon_{1}\right|=\left|\epsilon_{2}\right|=1\right)
$$

for $\gamma_{12}=\Xi_{1}=\Xi$. This is a finite group of orthogonal transformations $J$ in the space of the four components $X_{0}, X_{1}, X_{2}, X_{3}$ of the variable quaternion $\Xi$. Denote by $\Xi J$ the transform of $\Xi$ by $J$. The simplest way of ascertaining a fundamental domain for this group $\{J\}$ is as follows: One chooses a point $\Xi=\mathrm{A}$ which differs from all its transforms A $J$ ( $J \neq$ identity). The fundamental domain consists of all points $\Xi$ which are nearer to the center $\mathbf{A}$ than to the other equivalent centers $\mathrm{A} J$ and is thus characterized by the inequalities

$$
\Xi \cdot \overline{\mathrm{A}-\mathrm{A} J}+(\mathrm{A}-\mathrm{A} J) \cdot \bar{\Xi} \geqq 0 .
$$

Fortunately these are linear inequalities, namely of the form

$$
a_{0} X_{0}+a_{1} X_{1}+a_{2} X_{2}+a_{3} X_{3} \geqq 0
$$

$\left(a_{0}, a_{1}, a_{2}, a_{3}\right.$ being the components of $\left.\mathrm{A}-\mathrm{A} J\right)$. After having done this we limit ourselves to those operations $J_{\Lambda}$ which leave $\Xi_{1}$ unchanged. They form a subgroup and we study its influence upon $\Xi_{2}, \cdots, \Xi_{n-1}$. The next step would consist in singling out $\Xi_{2}$. By induction we thus obtain a finite number of subsidiary linear inequalities each concerned with the four components of one of the variables $\gamma_{12}, \cdots, \gamma_{n-1, n}$ only, and by them we define the fundamental domain $G_{\Lambda}{ }^{\prime}$ in $G_{\Lambda}$ for the group $\left\{J_{\Lambda}\right\}$.

I set little store by this whittling down of $G_{\Lambda}$ to $G_{\Lambda}{ }^{\prime}$. It seems less artificial to operate with the whole cells $Z$; in doing so one has to keep in mind that the modular group in its influence upon $Z$ matters only modulo $\left\{J_{Z}\right\}$.

Institute For Advanced Study,

Princeton, N. J. 